Surveys in Differential Geometry XV

\title{
Eta Forms and the Odd Pseudodifferential Families Index
}

\author{
Richard Melrose and Frédéric Rochon
}

\begin{abstract}
Let $A(t)$ be an elliptic, product-type suspended (which is to say parameter-dependant in a symbolic way) family of pseudodifferential operators on the fibres of a fibration $\phi$ with base $Y$. The standard example is $A+i t$ where $A$ is a family, in the usual sense, of first order, self-adjoint and elliptic pseudodifferential operators and $t \in \mathbb{R}$ is the 'suspending' parameter. Let $\pi_{\mathcal{A}}: \mathcal{A}(\phi) \longrightarrow Y$ be the infinite-dimensional bundle with fibre at $y \in Y$ consisting of the Schwartz-smoothing perturbations, $q$, making $A_{y}(t)+q(t)$ invertible for all $t \in \mathbb{R}$. The total eta form, $\eta_{\mathcal{A}}$, as described here, is an even form on $\mathcal{A}(\phi)$ which has basic differential which is an explicit representative of the odd Chern character of the index of the family:

$$
d \eta_{\mathcal{A}}=\pi_{\mathcal{A}}^{*} \gamma_{A}, \quad \operatorname{Ch}(\operatorname{ind}(A))=\left[\gamma_{A}\right] \in H^{\text {odd }}(Y) .
$$

The 1-form part of this identity may be interpreted in terms of the $\tau$ invariant (exponentiated eta invariant) as the determinant of the family. The 2-form part of the eta form may be interpreted as a B-field on the K-theory gerbe for the family $A$ with $(*)$ giving the 'curving' as the 3-form part of the Chern character of the index. We also give 'universal' versions of these constructions over a classifying space for odd K-theory. For Dirac-type operators, we relate $\eta_{\mathcal{A}}$ with the Bismut-Cheeger eta form.
\end{abstract}

\section{Introduction}

Eta forms, starting with the eta invariant itself, appear as the boundary terms in the index formula for Dirac operators $[\mathbf{2}],[\mathbf{5}],[\mathbf{4}],[\mathbf{2 1}],[\mathbf{2 2}]$. One aim of the present paper is to show that, with the freedom gained by

The research of the first author was partially supported by the National Science Foundation under grant DMS-0408993; the second author was supported by a NSERC discovery grant.

(C)2011 International Press 
working in the more general context of families of pseudodifferential operators, these forms appear as universal transgression, or connection, forms for the cohomology class of the index. That these forms arise in the treatment of boundary problems corresponds to the fact that boundary conditions amount to the explicit inversion of a suspended (or model) problem on the boundary. The odd index of the boundary family is trivial and the eta form is an explicit trivialization of it in cohomology. To keep the discussion within bounds we work here primarily in the 'odd' setting of a family of self-adjoint elliptic pseudodifferential operators, taken to be of order 1, on the fibres of a fibration of compact manifolds

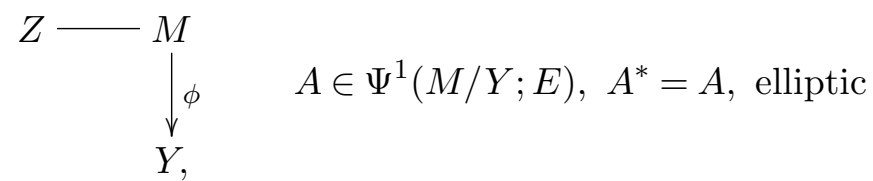

where a smooth, positive, fibre density on $M$ and a Hermitian inner product on the bundle have been chosen to define the adjoint. A similar discussion is possible in the more usual 'even' case.

From the fibration and pseudodifferential family an infinite-dimensional bundle of principal spaces, $\mathcal{A}(\phi)$, of invertible perturbations on each fibre, can be constructed:

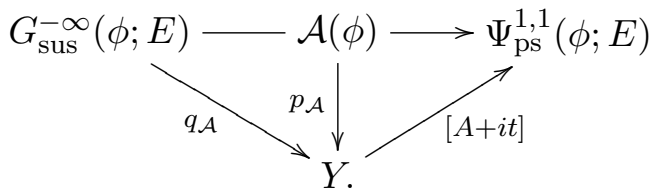

The vertical map here does not correspond to a principal bundle in the conventional sense since it has a non-constant bundle of structure groups, $G_{\text {sus }}^{-\infty}(\phi ; E)$, with fibre consisting of the invertible suspended smoothing perturbations of the identity on the corresponding fibre of $\phi$. The individual groups in this bundle are flat pointed loop groups and hence are classifying for even K-theory. Despite the twisting by fibre diffeomorphisms, the homotopy group $\Pi_{0}\left(G_{\text {sus }}^{-\infty}(M / Y ; E)\right)$, where $G_{\text {sus }}^{-\infty}(M / Y ; E)$ is the space of global smooth sections of $G_{\text {sus }}^{-\infty}(\phi ; E)$, is canonically identified with $\mathrm{K}^{0}(Y)$, see for instance $[\mathbf{3 0}]$. In this sense $G_{\text {sus }}^{-\infty}(\phi ; E)$ is a 'classifying bundle' for the K-theory of $Y$.

Throughout this paper we use notation such as $\mathcal{B}(\phi)$ for the total space of a bundle over $Y$ associated with a given fibration (1) and $\mathcal{B}(M / Y)$ for the corresponding space of global sections of the bundle. Thus, on the right in $(2), \Psi_{\mathrm{ps}}^{1,1}(\phi ; E)$ is the space of product-type suspended pseudodifferential operators on the fibres of $\phi$ (and acting on sections of the bundle $E$ ) an element of $\Psi_{\mathrm{ps}}^{1,1}(\phi, E)$ is thus a family of pseudodifferential operators acting on smooth sections of $E$ on the fibre above a point $y \in Y$ where the parameter in the family is in $\mathbb{R}$ (the suspension variable $t$ ) with 'product 
symbolic' dependence on this parameter (as indicated by the suffix ps). In the diagram above, $A+i t$ is such a family, although we consider a more general situation in the body of the paper.

On the total space of the structure bundle in (2) there is a deRham form, $\mathrm{Ch}_{\text {even }}$, representing the even Chern character, i.e. which pulls back under any section to a representative of the Chern character of the K-class defined by that section. The eta form, $\eta_{\mathcal{A}}$, in this setting is a form on $\mathcal{A}(\phi)$, defined by regularization of the formula for the Chern character on the structure bundle (see (6.4) and (6.6)). Under the action of a section of the structure bundle, this eta form shifts by the pull back of the Chern character up to an exact term

$$
\begin{gathered}
\alpha: G_{\mathrm{sus}}^{-\infty}(M / Y ; E) \times_{Y} \mathcal{A}(\phi) \longrightarrow \mathcal{A}(\phi), \alpha^{*} \eta_{\mathcal{A}}=\operatorname{pr}_{2}^{*} \eta_{\mathcal{A}}+\operatorname{pr}_{1}^{*} \mathrm{Ch}_{\mathrm{even}}+d \gamma \\
\gamma \text { a smooth form on } G_{\mathrm{sus}}^{-\infty}(M / Y ; E) \times_{Y} \mathcal{A}(\phi), \\
\text { where } \operatorname{pr}_{1}: G_{\mathrm{sus}}^{-\infty}(M / Y ; E) \times_{Y} \mathcal{A}(\phi) \longrightarrow G_{\mathrm{sus}}^{-\infty}(M / Y ; E) \text { and } \\
\operatorname{pr}_{2}: G_{\mathrm{sus}}^{-\infty}(M / Y ; E) \times_{Y} \mathcal{A}(\phi) \longrightarrow \mathcal{A}(\phi)
\end{gathered}
$$

are the natural projections. The central result below is:

THEOREM 1. The eta form, $\eta_{\mathcal{A}}$, on $\mathcal{A}(\phi)$ has basic differential representing the odd Chern character of the index bundle of the given family $A$ in (1)

$d \eta_{\mathcal{A}}=p_{\mathcal{A}}^{*} \gamma_{A}, \gamma_{A} \in \mathcal{C}^{\infty}\left(Y ; \Lambda^{o d d}\right), d \gamma_{A}=0, \mathrm{Ch}_{\text {odd }}(\operatorname{ind}(A))=\left[\gamma_{A}\right] \in H^{o d d}(Y)$

Once a choice of connection is made, the form $\gamma_{\mathcal{A}}$, which can be written explicitly in terms of the formal trace of [20] (see (6.13) below), gives a representative of the Chern character of the index class. For the particular case of families of Dirac operators associated to a pseudodifferential bundle, Paycha and Mickelsson, in [28], obtained a related representative of the Chern class using the Wodzicki residue instead of the formal trace.

To prove Theorem 1 we use the smooth delooping sequence for the fibration, which is the top row in the diagram

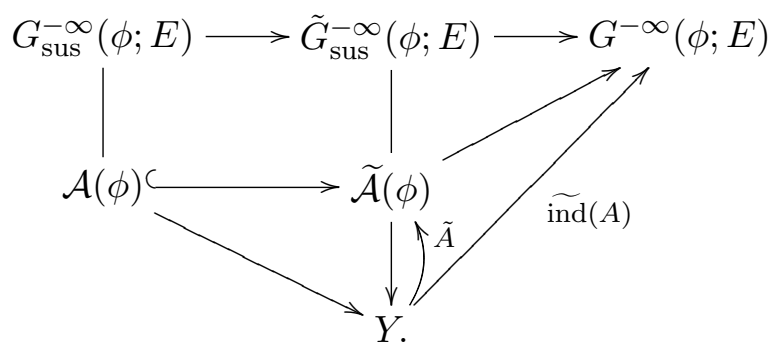


Here $\widetilde{\mathcal{A}}(\phi)$ is an extension of $\mathcal{A}(\phi)$ to a bundle of principal spaces (in the same sense as for $\mathcal{A})$ with bundle of structure groups, $\tilde{G}_{\text {sus }}^{-\infty}(\phi ; E)$, the halfopen (smooth-flat) loop group bundle. This has contractible fibres and hence $\widetilde{\mathcal{A}}(\phi)$ has a section $\widetilde{A}$ as indicated in (5). Taking the quotient by the original structure group, this projects to a section, $\widetilde{\operatorname{ind}}(A)$, of $G^{-\infty}(\phi ; E)$ with homotopy class giving (minus) the index in $\mathrm{K}^{1}(Y)$ of the family. Ultimately, (4) follows from the fact that there is a corresponding multiplicativity formula linking $\eta_{\mathcal{A}}$ to an analogous form, $\widetilde{\eta}$. Thus $\widetilde{\eta}$ is a universal transgression form for the delooping sequence, in that it restricts to the Chern character on $G_{\text {sus }}^{-\infty}(\phi ; E)$ and $d \widetilde{\eta}$ is basic; it is the pull-back of the odd Chern character $\mathrm{Ch}_{\text {odd }}$ on $G^{-\infty}(\phi ; E)$.

In $\S 1$ the smooth delooping sequence for K-theory is described. The universal Chern forms on the odd and even classifying spaces are constructed in $\S 2$; the regularization to a universal eta form on the half-open loop group is carried out in $\S 3$. The constructions of Chern forms is extended to the classifying bundle given by a fibration in $\S 4$. The bundle of invertible perturbations for a self-adjoint elliptic family, or more generally an elliptic family of product-type suspended operators, is introduced in $\S 5$ and in $\S 6$ the eta forms are generalized to this case and further extended in $\S 7$. The index formula, Theorem 1 , is proved in $\S 8$. The realization of the exponentiated eta invariant, the $\tau$-invariant, as a determinant is discussed in $\S 9$ and the adiabatic determinant of a doubly-suspended family is discussed in $\S 10$. This is used to construct a smooth and primitive form of the determinant line bundle over the even classifying space in $\S 11$. The K-theory gerbe is realized as a bundle gerbe in the sense of Murray [26] in $\S 12$ and the geometric version of this gerbe for an elliptic family is described in $\S 13$. Finally, in $\S 14$, we discuss the relationship between the eta forms as introduced here and the eta forms of Bismut-Cheeger [3].

\section{Delooping sequence}

We first consider the 'universal' case with constructions directly over classifying spaces. Despite the infinite-dimensional base, this setting is a little simpler than the geometric case of a fibration since there is no twisting by diffeomorphisms. Let $Z$ be a compact manifold with $\operatorname{dim} Z>0$ and let $E \longrightarrow Z$ be a complex vector bundle over it. The algebra of smoothing operators on sections of $E$ is

$$
\Psi^{-\infty}(Z ; E)=\mathcal{C}^{\infty}\left(Z^{2} ; \operatorname{Hom}(E) \otimes \Omega_{R}\right)
$$

where $\Omega_{R}=\pi_{R}^{*} \Omega$ is the pull-back of the density bundle by the projection $\pi_{R}: Z \times Z \longrightarrow Z$ onto the right factor and $\operatorname{Hom}(E)=\pi_{R}^{*} E^{\prime} \otimes \pi_{L}^{*} E$ with $\pi_{L}: Z \times Z \longrightarrow Z$ the projection onto the left factor. The product is given by the integral

$$
(A \circ B)\left(z, z^{\prime}\right)=\int_{Z} A\left(z, z^{\prime \prime}\right) B\left(z^{\prime \prime}, z^{\prime}\right)
$$


The topological group

$$
G^{-\infty}(Z ; E)=\left\{A \in \Psi^{-\infty}(Z ; E) ; \exists(\mathrm{Id}+A)^{-1}=\mathrm{Id}+B, B \in \Psi^{-\infty}(Z ; E)\right\}
$$

is an open dense subset and is classifying for odd K-theory. The 'suspended' (or flat-pointed loop) group

$$
G_{\text {sus }}^{-\infty}(Z ; E)=\left\{A \in \mathcal{S}\left(\mathbb{R}_{\tau} \times Z^{2} ; \operatorname{Hom}(E) \otimes \Omega_{R}\right) ; A(\tau) \in G^{-\infty}(Z ; E)\right\}
$$

is therefore classifying for even K-theory. It is an open (and dense) subspace of the Schwartz functions on $\mathbb{R}$ with values in $\Psi^{-\infty}(Z ; E)$.

Thus, for any other manifold $X$, the sets of equivalence classes of (smooth) maps reducing to the identity outside a compact set under (smooth) homotopy through such maps are the K-groups:

$$
\begin{aligned}
& \mathrm{K}_{\mathrm{c}}^{1}(X)=\left\{f \in \mathcal{C}^{\infty}\left(X ; G^{-\infty}(Z ; E)\right) ; f=\mathrm{Id} \text { on } X \backslash K, K \Subset X\right\} / \sim, \\
& \mathrm{K}_{\mathrm{c}}^{0}(X)=\left\{f \in \mathcal{C}^{\infty}\left(X ; G_{\text {sus }}^{-\infty}(Z ; E)\right) ; f=\mathrm{Id} \text { on } X \backslash K, K \Subset X\right\} / \sim .
\end{aligned}
$$

By definition, Schwartz functions are 'flat at infinity' and we introduce a larger space of functions which are Schwartz at $-\infty$ but more generally 'flat to a constant' at $+\infty$ and the corresponding group

$$
\begin{aligned}
\tilde{G}_{\mathrm{sus}}^{-\infty}(Z ; E)= & \left\{A \in \mathcal{C}^{\infty}\left(\mathbb{R}_{\tau} \times Z^{2} ; \operatorname{Hom}(E) \otimes \Omega_{R}\right) ; \lim _{\tau \rightarrow-\infty} A(\tau)=0,\right. \\
& \frac{d A(\tau)}{d \tau} \in \mathcal{S}\left(\mathbb{R}_{\tau} \times Z^{2} ; \operatorname{Hom}(E) \otimes \Omega_{R}\right), \\
& \left.A(\tau) \in G^{-\infty}(Z ; E) \forall \tau \in[-\infty, \infty]\right\}
\end{aligned}
$$

Thus $A$ can be recovered from its derivative,

$$
A(\tau)=\int_{-\infty}^{\tau} \frac{d A(s)}{d s} d s
$$

Moreover, there is a well-defined map 'restriction to $\tau=\infty$ ',

$$
R_{\infty}: \tilde{G}_{\text {sus }}^{-\infty}(Z ; E) \longrightarrow G^{-\infty}(Z ; E)
$$

which is surjective since $G^{-\infty}(Z ; E)$ is connected and a general curve between two points can be smoothed and flattened at the ends.

The delooping sequence in the present context is the short exact sequence of groups

$$
G_{\mathrm{sus}}^{-\infty}(Z ; E) \stackrel{\iota}{\longrightarrow} \tilde{G}_{\mathrm{sus}}^{-\infty}(Z ; E) \stackrel{R_{\infty}}{\longrightarrow} G^{-\infty}(Z ; E)
$$

where the map to the quotient group is explicitly given by (1.6) and the flatness of the paths at $+\infty$ ensures exactness in the middle.

Lemma 1. The group $\tilde{G}_{\text {sus }}^{-\infty}(Z ; E)$ is contractible. 
Proof. It is only the 'flatness at infinity' of the elements of $\tilde{G}_{\text {sus }}^{-\infty}(Z ; E)$ that distinguishes this result from the standard contractibility, by shortening the curve, of the pointed path space of a group. To maintain this condition during the contraction, first identify $(-\infty, \infty)$ by radial compactification with the interior of $[0,1]$. Since the singularities in the compactification are swamped by the rapid vanishing of the derivatives at the end points, this gives the alternative description of the group as

$$
\begin{aligned}
\tilde{G}_{\mathrm{sus}}^{-\infty}(Z ; E)=\left\{a \in \mathcal{C}^{\infty}\left([0,1]_{x} ; G^{-\infty}(Z ; E)\right) ; \frac{d a}{d x} \in \dot{\mathcal{C}}^{\infty}([0,1] ;\right. \\
\\
\left.\left.\Psi^{-\infty}(Z ; E)\right), a(0)=0\right\}
\end{aligned}
$$

where $\dot{\mathcal{C}}^{\infty}\left([0,1] ; \Psi^{-\infty}(Z ; E)\right)$ is the space of smooth functions vanishing together with all their derivatives at $x=0$ and $x=1$.

Now, let $\rho:[0,1] \longrightarrow[0,1]$ be a smooth function with $\rho(0)=0$ and $\rho(x)=1$ near $x=1$ and consider the homotopy

$$
\psi_{t}(x)= \begin{cases}2 t \rho(x), & t \in\left[0, \frac{1}{2}\right], \\ \rho(x)+(2 t-1)(x-\rho(x)), & t \in\left[\frac{1}{2}, 1\right],\end{cases}
$$

between the constant map, $\psi_{0}(x)$, and the identity map $\psi_{1}(x)=x$. Note that $\psi_{t}(1)=1$ for $\frac{1}{2} \leq t \leq 1$ and $\psi_{t}$ is flat at 1 for $0 \leq t \leq \frac{1}{2}$. It follows that the composite $f\left(\psi_{t}(x)\right)$ with $f \in \mathcal{C}^{\infty}([0,1])$ is flat at $x=1$ for all $t$ if $f$ is flat at $x=1$. Thus composition

$$
\Psi_{t}: \tilde{G}_{\text {sus }}^{-\infty}(Z ; E) \ni a \longmapsto a \circ \psi_{t} \in \tilde{G}_{\text {sus }}^{-\infty}(Z ; E)
$$

gives the desired deformation retraction to the identity element.

This argument is not limited to this particular group and holds in greater generality.

\section{Universal Chern forms}

The group $G^{-\infty}(Z ; E)$, identified as an open dense subset of $\Psi^{-\infty}(Z ; E)$, is an infinite dimensional manifold modelled on the Fréchet space $\mathcal{C}^{\infty}\left(Z^{2}\right.$; $\left.\operatorname{Hom}(E) \otimes \Omega_{R}\right)$. We shall fix the space of smooth functions on $G^{-\infty}(Z ; E)$ and more generally the smooth sections of form bundles and other tensor bundles.

First, it is natural to identify the tangent space at any point with the linear space in which the group is embedded. Then the cotangent space can be identified with its dual, $\mathcal{C}^{-\infty}\left(Z^{2} ; \operatorname{Hom}\left(E^{\prime}\right) \otimes \Omega_{L}\right)$, the space of distributional sections, where $\Omega_{L}$ is the left density bundle. Thus

$$
T_{a}^{*} G^{-\infty}(Z ; E)=\mathcal{C}^{-\infty}\left(Z^{2} ; \operatorname{Hom}\left(E^{\prime}\right) \otimes \Omega_{L}\right)
$$


with the duality between smooth tangent and cotangent fibres given by distributional pairing. This can be written formally as bundle pairing followed by integration

$$
\begin{gathered}
T_{a}^{*} G^{-\infty}(Z ; E) \times T_{a} G^{-\infty}(Z ; E) \ni(\alpha, B) \longrightarrow \alpha \cdot B \in \mathbb{C} \\
\alpha \cdot B=\int_{Z^{2}} \alpha\left(z, z^{\prime}\right) B\left(z, z^{\prime}\right)
\end{gathered}
$$

Having defined the tangent and cotangent fibres at each point, the fibres of the cotensor bundles are interpreted as completed tensor products. Thus

$$
\left(T^{*}\right)_{a}^{\otimes k}=\mathcal{C}^{-\infty}\left(Z^{2 k} ; \bigotimes_{j} \pi_{j}^{*} \operatorname{Hom}\left(E^{\prime}\right) \otimes \Omega_{k L}\right)
$$

where $\Omega_{k L}$ is the tensor product of the (trivial) real line bundles on each left factor of all the pairs and the homomorphism bundle is lifted from each pair of factors.

Since $G^{-\infty}(Z ; E)$ is a metric space with the topology induced from $\Psi^{-\infty}(Z ; E)$, continuity for functions is immediately defined. More generally, continuity for sections of any of the tensor bundles is defined by insisting that a $k$-cotensor field should be a continuous map from the metric space $G^{-\infty}(Z ; E)$ (or indeed any subset of it) into the distributional space (2.3) in the strong sense that it should map locally into some fixed Sobolev, hence Hilbert, space

$$
H^{-N}\left(Z^{2 k} ; \bigotimes_{j} \pi_{j}^{*} \operatorname{Hom}\left(E^{\prime}\right) \otimes \Omega_{k L}\right)
$$

and should be continuous for the metric topologies. The meaning of directional derivatives is then clear. For a map to be $C^{1}$, we insist that all directional derivatives exist at each point, that they are jointly defined by an element of the next higher tensor space, i.e. distribution in two more variables, and that the resulting section of this tensor bundle is also continuous. Then infinite differentiability is defined by iteration.

The form bundles are defined, as usual, as the totally antisymmetric parts of the corresponding cotensor bundles. Smoothness as a form is smoothness as a cotensor field. The deRham differential is the map from smooth $k$-forms to smooth $(k+1)$-forms given in the usual way by differentiation followed by antisymmetrization.

If $F: G^{-\infty}(Z ; E) \longrightarrow \mathbb{C}$ is smooth and $b \in G^{-\infty}(Z ; E)$ then $L(b)^{*} F(a)=$ $F(b a)$ is also smooth, as is $R(b)^{*} F$ defined by $R(b)^{*} F(a)=F\left(a b^{-1}\right)$. Thus $G^{-\infty}(Z ; E)$ acts on its space of smooth functions, as in the setting of finite dimensional Lie groups. These actions extend to cotensor fields and hence to forms. 
The universal odd Chern character is given by a slight reinterpretation of the standard finite-dimensional formula

$$
\begin{aligned}
\operatorname{Ch}_{\text {odd }}(a)= & \frac{1}{2 \pi i} \operatorname{Tr}\left(\int_{0}^{1} a^{-1} d a \exp \left(\frac{t(1-t)\left(a^{-1} d a\right)^{2}}{2 \pi i}\right) d t\right) \\
& \in \mathcal{C}^{\infty}\left(G^{-\infty}(Z ; E) ; \Lambda^{\text {odd }}\right) .
\end{aligned}
$$

Namely expanding out the exponential in formal power series and carrying out the resulting integrals reduces this to an infinite sum

$$
\mathrm{Ch}_{\text {odd }}(a)=\sum_{k=0}^{\infty} c_{k} \operatorname{Tr}\left(\left(a^{-1} d a\right)^{2 k+1}\right), \quad c_{k}=\frac{1}{(2 \pi i)^{k+1}} \frac{k !}{(2 k+1) !} .
$$

Here, each $d a$ is the identification of the tangent space at $a$ with $\Psi^{-\infty}(Z ; E)$ - so can be thought of as the differential of the identity. Thus, for any $2 k+1$ elements $b_{j} \in \Psi^{-\infty}(Z ; E)$, the evaluation on $\left(T_{a}\right)^{\otimes(2 k+1)}$ of an individual term is

$$
\begin{aligned}
\operatorname{Tr} & \left(\left(a^{-1} d a\right)^{2 k+1}\right)\left(b_{1}, \ldots, b_{2 k+1}\right) \\
& =\sum_{\sigma}(-1)^{\operatorname{sign}(\sigma)} \operatorname{Tr}\left(a^{-1} b_{\sigma(1)} a^{-1} b_{\sigma(2)} \ldots a^{-1} b_{\sigma(2 k+1)}\right) .
\end{aligned}
$$

The trace is well defined since the product is an element of $\Psi^{-\infty}(Z ; E)$. It is also defined at each point by a distribution, as required above, and the same is true of all derivatives. Namely at each point the distribution defining this form is just the total antisymmetrization (of variables in pairs) of

$$
A\left(z_{2 k+1}^{\prime}, z_{1}\right) A\left(z_{1}^{\prime}, z_{2}\right) A\left(z_{2}^{\prime}, z_{3}\right) \ldots A\left(z_{2 k}^{\prime}, z_{2 k+1}\right)
$$

where $A$ is the Schwartz kernel of $a^{-1}$. Note that while this $i s$ smooth in the sense described above, the kernel representing the form at a given point is not a smooth function because of the presence of the identity factors in the operators. Due to the identity

$$
\frac{d}{d t} a_{t}^{-1}=-a^{-1} \frac{d a_{t}}{d t} a^{-1}
$$

differentiation gives a similar form, but with less symmetrization, with respect to parameters. Thus (2.6) defines a form in each odd degree.

As a result of antisymmetrization the form corresponding to (2.6) for an even power is identically zero. Moreover the computation of the deRham differential, based on the identities (2.8), $d^{2} a=0$ and $d\left(a^{-1} d a a^{-1}\right)=0$ yields

$$
d \operatorname{Tr}\left(\left(a^{-1} d a\right)^{2 k+1}\right)=-\operatorname{Tr}\left(\left(a^{-1} d a\right)^{2 k+2}\right)=0 \Longrightarrow d \mathrm{Ch}_{\mathrm{odd}}=0
$$

globally on $G^{-\infty}(Z ; E)$. The Chern character (2.4) is universal in the sense that if $f: X \longrightarrow G^{-\infty}(Z ; E)$ is any smooth map from a compact manifold $X$, then

$$
\left[f^{*} \mathrm{Ch}_{\text {odd }}\right]=\mathrm{Ch}_{\text {odd }}([f]) \in H^{\text {odd }}(X ; \mathbb{C})
$$


represents the odd Chern character of the $K$-class defined by the homotopy class $[f]$ of $f$.

The abelian group structure on $\mathrm{K}^{1}(X)$ is derived from the non-abelian group structure on $G^{-\infty}(Z ; E)$ and in particular the linearity of the odd Chern character is a consequence of the following result. Here we say that a form on a product of two (infinite-dimensional) manifolds $M_{1} \times M_{2}$ 'has no pure terms' if it vanishes when restricted to $\left\{p_{1}\right\} \times M_{2}$ or $M_{1} \times\left\{p_{2}\right\}$ for any points $p_{1} \in M_{1}$ or $p_{2} \in M_{2}$.

Proposition 1. There is a smooth form $\delta_{\text {even }}$ on $G^{-\infty}(Z ; E) \times G^{-\infty}$ $(Z ; E)$ of even degree which has no pure terms, vanishes when pulled back to the 'product diagonal' $\left\{\left(a, a^{-1}\right)\right\}$ and is such that in terms of pull-back under the product map and two projections:

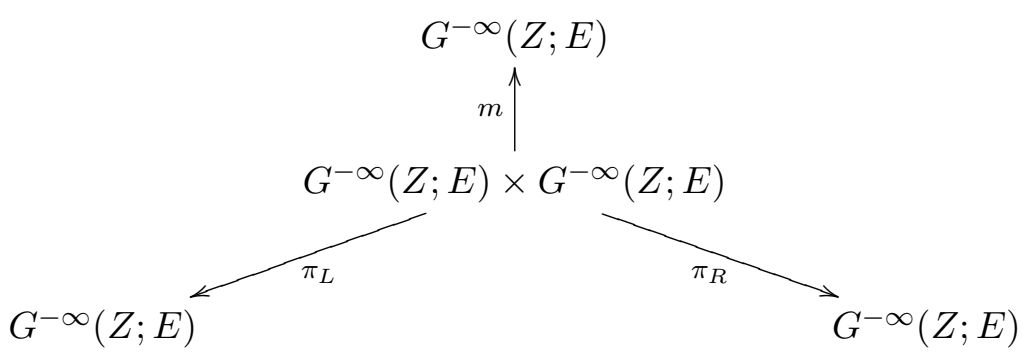

the form in (2.4) satisfies

$$
m^{*} \mathrm{Ch}_{o d d}=\pi_{L}^{*} \mathrm{Ch}_{o d d}+\pi_{R}^{*} \mathrm{Ch}_{o d d}+d \delta_{\text {even }} .
$$

Proof. For any two bundles the group $G^{-\infty}(Z ; E) \oplus G^{-\infty}(Z ; F)$ can be identified as the diagonal subgroup of $G^{-\infty}(Z ; E \oplus F)$ and the Chern form restricted to this subgroup clearly splits as the direct sum. So, to prove (2.12) we work on $E \oplus E$ and take a homotopy which connects $a b$ acting on the left factor of $E$, so as $a b \oplus \operatorname{Id}$ on $E \oplus E$, with $a \oplus b$ acting on $E \oplus E$. This can be constructed in terms of a rotation between the two factors. Thus

$$
M(t)=\left(\begin{array}{cc}
\cos t & \sin t \\
-\sin t & \cos t
\end{array}\right), \quad t \in[0, \pi / 2]
$$

is such that

$$
\begin{aligned}
& B(t)=M^{-1}(t)\left(\begin{array}{cc}
b & 0 \\
0 & \text { Id }
\end{array}\right) M(t) \quad \text { satisfies } B(0)=\left(\begin{array}{cc}
b & 0 \\
0 & \text { Id }
\end{array}\right), \\
& B(\pi / 2)=\left(\begin{array}{cc}
\text { Id } & 0 \\
0 & b
\end{array}\right) .
\end{aligned}
$$

Using this family, consider the map

$$
H:[0,1] \times G^{-\infty}(Z ; E) \times G^{-\infty}(Z ; E) \longmapsto A(0) B(t) \in G^{-\infty}(Z ; E \oplus E) .
$$


It follows that the form $\alpha=H^{*} \mathrm{Ch}_{\text {odd }}$ is a closed form on the product and hence decomposing in terms of the factor $[0,1]$,

$$
\alpha=d t \wedge \alpha_{1}(t)+\alpha_{2}(t)
$$

where the $\alpha_{i}$ are smooth 1-parameter families of forms on $G^{-\infty}(Z ; E) \times$ $G^{-\infty}(Z ; E)$

$$
d \alpha_{2}=0, \quad d \alpha_{1}=\frac{\partial}{\partial t} \alpha_{2}
$$

where $d$ is now the deRham differential on $G^{-\infty}(Z ; E) \times G^{-\infty}(Z ; E)$. Thus, setting

$$
\delta_{\text {even }}=-\int_{0}^{\pi / 2} \alpha_{1}(t) d t
$$

(2.12) follows.

Now, if $a$ is held constant, $H^{*} \mathrm{Ch}_{\text {odd }}$ is independent of $a$ and reduces to the Chern character for $B(t)$. It follows that the individual terms in $\alpha_{1}$ are multiples of

$$
\begin{gathered}
\operatorname{Tr}\left(\left(\begin{array}{cc}
\operatorname{Id} & 0 \\
0 & 0
\end{array}\right)\left(\frac{\partial M(t)}{\partial t} M^{-1}(t)\left((d b) b^{-1}\right)^{2 k}\right)\right. \\
\left.-\left(\begin{array}{cc}
\operatorname{Id} & 0 \\
0 & 0
\end{array}\right)\left(M^{-1}(t) \frac{\partial M(t)}{\partial t}\left(b^{-1} d b\right)^{2 k}\right)\right) .
\end{gathered}
$$

Since $\frac{\partial M(t)}{\partial t} M^{-1}(t)$ and $M^{-1}(t) \frac{\partial M(t)}{\partial t}$ are off-diagonal this vanishes. A similar argument applies if $b$ is held constant, so $\delta_{\text {even }}$ in (2.18) is without pure terms.

Under inversion, $a \longmapsto a^{-1}, \mathrm{Ch}_{\text {odd }}$ simply changes sign, so under the involution $I:(a, b) \longmapsto\left(b^{-1}, a^{-1}\right)$ both the left side and the two Chern terms, together, on the right change sign. Thus $\delta=\delta_{\text {even }}$ can be replaced by its odd part under this involution, $\frac{1}{2}\left(\delta-I^{*} \delta\right)$, which ensures that it vanishes when pulled back to the submanifold left invariant by $I$, namely $\left\{b=a^{-1}\right\}$. It still is without pure terms so the proposition is proved.

The discussion of the suspended group $G_{\text {sus }}^{-\infty}(Z ; E)$ is similar. Namely the tangent space is the space of Schwartz sections $\mathcal{S}\left(\mathbb{R} \times Z^{2} ; \operatorname{Hom}(E) \otimes\right.$ $\left.\Omega_{R}\right)$ which can be identified, by radial compactification of the line, with $\dot{\mathcal{C}}^{\infty}\left([-1,1] \times Z^{2} ; \operatorname{Hom}(E) \otimes \Omega_{R}\right) \subset \mathcal{C}^{\infty}\left([-1,1] \times Z^{2} ; \operatorname{Hom}(E) \otimes \Omega_{R}\right)$, consisting of the space of smooth sections on this manifold with boundary, vanishing to infinite order at both boundaries. The dual space is then the space of Schwartz distributions $\mathcal{S}^{\prime}\left(\mathbb{R} \times Z^{2} ; \operatorname{Hom}\left(E^{\prime}\right) \otimes \Omega_{L}\right)$, or in the compactified picture the space of extendible distributional sections. Apart from these minor alterations, the discussion proceeds as before and the even Chern forms, defined by pull-back and integration are

$$
\mathrm{Ch}_{\text {even }}=p_{*}\left(\mathrm{ev}^{*} \mathrm{Ch}_{\mathrm{odd}}\right) \in \mathcal{C}^{\infty}\left(G_{\text {sus }}^{-\infty}(Z ; E) ; \Lambda^{\mathrm{even}}\right)
$$


where

$$
\mathrm{ev}: \mathbb{R} \times G_{\text {sus }}^{-\infty}(Z ; E) \ni(s, A) \longmapsto A(s) \in G^{-\infty}(Z ; E)
$$

is the evaluation map and $p_{*}$ is the pushforward map along the fibres of the projection $p: \mathbb{R} \times G_{\text {sus }}^{-\infty}(Z ; E) \longrightarrow G_{\text {sus }}^{-\infty}(Z ; E)$ on the right factor. So, at least formally,

$$
\mathrm{Ch}_{\text {even }}=\frac{1}{2 \pi i} \int_{\mathbb{R}} \int_{0}^{1} \operatorname{Tr}\left(\left(a^{-1} d a\right) \exp \left(\frac{t(1-t)\left(a^{-1} d a\right)^{2}}{2 \pi i}\right)\right) d t
$$

where the outer integral mean integration with respect to $\tau$ of the coefficient of $d \tau$.

The analogue of Proposition 1 for the even Chern character follows from that result. Namely if we consider the corresponding product map, pointwise in the parameter, and projections:

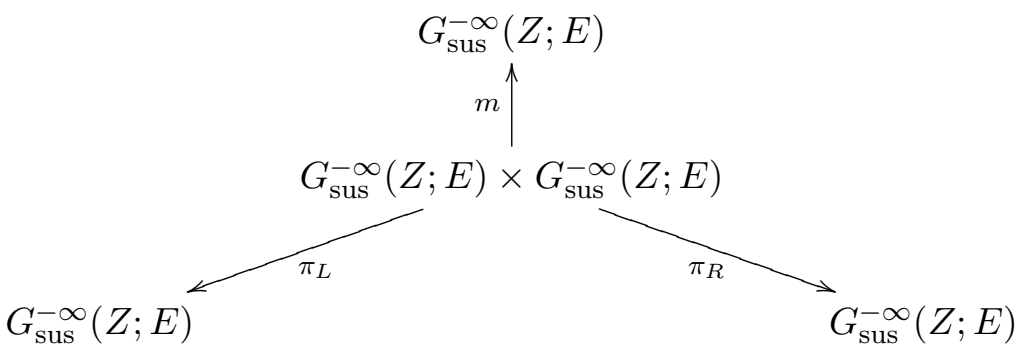

then there is a smooth form $\delta_{\text {odd }}$ on the product group such that

$$
m^{*} \mathrm{Ch}_{\text {even }}=\pi_{L}^{*} \mathrm{Ch}_{\text {even }}+\pi_{R}^{*} \mathrm{Ch}_{\text {even }}+d \delta_{\text {odd }} .
$$

This odd form can be constructed from $\delta_{\text {even }}$ using the pull-back and pushforward operations for the (product) evaluation map

$$
\begin{aligned}
\mathrm{Ev} & : \mathbb{R} \times G_{\text {sus }}^{-\infty}(Z ; E) \times G_{\text {sus }}^{-\infty}(Z ; E) \ni(\tau, a, b) \\
& \longrightarrow(a(\tau), b(\tau)) \in G^{-\infty}(Z ; E) \times G^{-\infty}(Z ; E)
\end{aligned}
$$

as

$$
\delta_{\text {odd }}=-\int_{\mathbb{R}} \delta^{\prime}(\tau) d \tau, \quad \mathrm{Ev}^{*} \delta_{\text {even }}=d \tau \wedge \delta^{\prime}(\tau)+\delta^{\prime \prime}(\tau) .
$$

Since the forms are Schwartz in the evaluation parameter, the additional term

$$
\int_{\mathbb{R}} \frac{\partial}{\partial \tau} \delta^{\prime \prime}(\tau)=0
$$

and (2.24) follows; note that it does not follow from the fact that $\delta_{\text {even }}$ has no pure terms that this is true of $\delta_{\text {odd }}$ - and it is not!

Smoothness of forms in the sense discussed above certainly implies that the pull-back of such a form to a finite dimensional manifold, under a smooth map $Y \longrightarrow G^{-\infty}(Z ; E)$ is smooth on $Y$ and closed if the form on $G^{-\infty}(Z ; E)$ is closed. Thus if $f: Y \longrightarrow G^{-\infty}(Z ; E)$ is a representative of $[f] \in \mathrm{K}^{1}(Y)$ then 
$f^{*} \mathrm{Ch}_{\text {odd }}$ is a sum of closed odd-degree forms on $Y$. The cohomology class is constant under homotopy of the map. Indeed, a homotopy between $f_{0}$ and $f_{1}$ is a map $F:[0,1]_{r} \times Y \longrightarrow G^{-\infty}(Z ; E)$. The fact that the Chern form pulls back to be closed shows that $F^{*} \mathrm{Ch}_{\text {odd }}$ is of the form

$$
\begin{aligned}
& A(r)+d r \wedge B(r), \quad d_{Y} A(r)=0, \quad \frac{d A(r)}{d r}=d_{Y} B(r) \\
& \Longrightarrow A(1)-A(0)=d \int_{0}^{1} B(r) d r .
\end{aligned}
$$

Thus cohomology classes in the even case are also homotopy invariant and these universal Chern forms define a map from K-theory to cohomology. This is the Chern character. The theorem of Atiyah and Hirzebruch shows that the combined even and odd Chern characters give a multiplicative isomorphism

$$
\mathrm{Ch}:\left(\mathrm{K}^{0}(X) \oplus \mathrm{K}^{1}(X)\right) \otimes \mathbb{C} \longrightarrow \mathrm{H}^{*}(X ; \mathbb{C}) .
$$

\section{Universal eta form}

As a link between the odd and even universal Chern characters defined above on the end groups in (1.7), we consider the corresponding eta form on $\tilde{G}_{\text {sus }}^{-\infty}(Z ; E)$. It has the same formal definition as the even Chern character but now lifted to the larger (and contractible) group. This consists of paths in $G^{-\infty}(Z ; E)$ so there is still an evaluation map

$$
\widetilde{\mathrm{Ev}}: \mathbb{R} \times \tilde{G}_{\mathrm{sus}}^{-\infty}(Z ; E) \ni(s, A) \longmapsto A(s) \in G^{-\infty}(Z ; E)
$$

just as in (2.21).

Definition 1. The universal eta form on $\tilde{G}_{\text {sus }}^{-\infty}(Z ; E)$ is defined as in (2.20) but interpreted on the group $\widetilde{G}_{\text {sus }}^{-\infty}(Z ; E)$ with the evaluation map (3.1)

$$
\tilde{\eta}=\tilde{p}_{*}\left(\widetilde{\mathrm{Ev}}^{*} \mathrm{Ch}_{\text {odd }}\right) \in \mathcal{C}^{\infty}\left(\widetilde{G}_{\text {sus }}^{-\infty}(Z ; E) ; \Lambda^{\text {even }}\right)
$$

and with $\tilde{p}_{*}$ the push-forward map corresponding to integration along the fibres of the projection $\tilde{p}: \mathbb{R} \times \widetilde{G}_{\text {sus }}^{-\infty}(Z ; E) \longrightarrow \widetilde{G}_{\text {sus }}^{-\infty}(Z ; E)$.

Integration on the fibres here is well defined since, in the integrand which is the contraction with $\partial / \partial \tau$ - necessarily one of the terms is differentiated with respect to the suspension parameter, which has the effect of removing the constant term at infinity. Thus the integral in (3.2) still converges rapidly.

If $X$ is a compact smooth manifold and if $a: X \longrightarrow \widetilde{G}^{-\infty}(Z ; E)$ is a smooth map, the associated eta form is

$$
\eta(a)=a^{*} \tilde{\eta}
$$


Now, consider the diagram analogous to (2.23) but for the extended group, and hence with an additional map corresponding to restriction to $t=\infty$ in each factor:

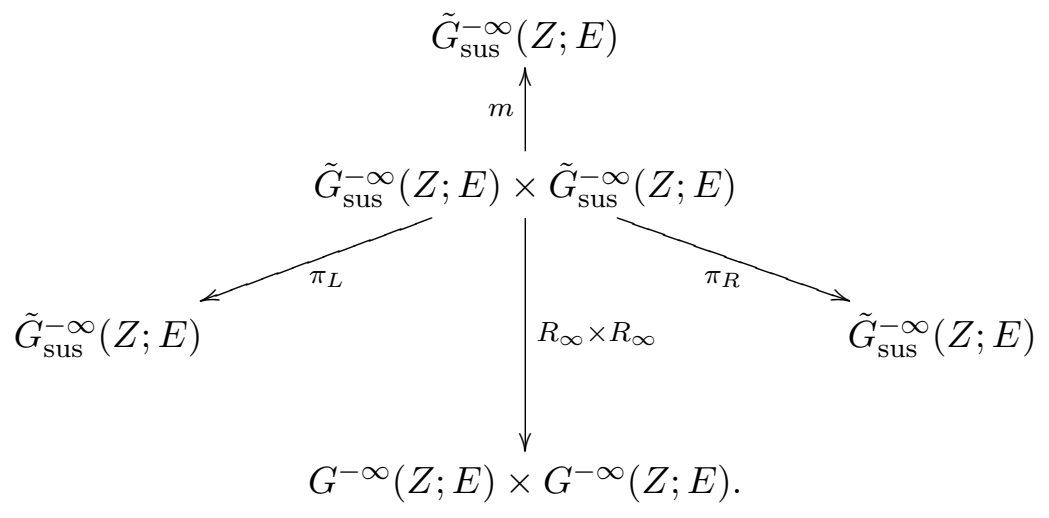

Proposition 2. The eta form in (3.2) restricts to $\mathrm{Ch}_{\text {even }}$ on $G_{\text {sus }}^{-\infty}$ $(Z ; E)$, satisfies the identity

$$
m^{*} \tilde{\eta}=\pi_{L}^{*} \tilde{\eta}+\pi_{R}^{*} \tilde{\eta}+d\left(\tilde{\delta}_{\text {odd }}\right)+\left(R_{\infty} \times R_{\infty}\right)^{*} \delta_{\text {even }}
$$

where $\tilde{\delta}_{\text {odd }}$ is a smooth form on $\tilde{G}_{\text {sus }}^{-\infty}(Z ; E) \times \tilde{G}_{\text {sus }}^{-\infty}(Z ; E)$ which restricts to $\delta_{\text {odd }}$ on $G_{\text {sus }}^{-\infty}(Z ; E) \times G_{\text {sus }}^{-\infty}(Z ; E)$ and moreover $\tilde{\eta}$ has basic differential

$$
d \tilde{\eta}=R_{\infty}^{*} \mathrm{Ch}_{\text {odd }}
$$

where $R_{\infty}$ is the quotient map in (1.7).

Proof. To compute the differential of the eta form, write the pull-back under $\widetilde{\mathrm{Ev}}$ as in $(2.28)$ :

$$
\widetilde{\mathrm{Ev}}^{*} \mathrm{Ch}_{\mathrm{odd}}=A(\tau)+d \tau \wedge B(\tau) \Longrightarrow d B(\tau)=\frac{d A(\tau)}{d \tau}
$$

Since

$$
\tilde{\eta}=\int_{\mathbb{R}} B(\tau), \quad d \tilde{\eta}=\int_{\mathbb{R}} d B(\tau) d \tau=\int_{\mathbb{R}} \frac{d A(\tau)}{d \tau} d \tau=A(\infty)=R_{\infty}^{*} \mathrm{Ch}_{\mathrm{odd}} .
$$

This proves (3.6).

Similarly, as in the proof of (2.24), pulling back the corresponding additivity formula, (2.12), for the odd Chern character gives (3.5) with the additional term arising from the integral which vanishes as in (2.27) on the suspended subgroup. 


\section{Geometric Chern forms}

Next we pass to a discussion of the 'geometric case'. Fix a connection on the fibration (1). That is, choose a smooth splitting

$$
T M=T^{H} M \oplus T(M / Y)
$$

where the subbundle $T^{H} M$ is necessarily isomorphic to $\phi^{*} T Y$. Also choose a connection $\nabla^{E}$ on the complex vector bundle $E \longrightarrow M$. Consider the infinitedimensional bundle

$$
\mathcal{C}^{\infty}(\phi ; E) \longrightarrow Y
$$

which has fibre $\mathcal{C}^{\infty}\left(Z_{y} ; E_{y}\right), Z_{y}=\phi^{-1}(y), E_{y}=\left.E\right|_{Z_{y}}$ at $y \in Y$ and space of smooth global sections written $\mathcal{C}^{\infty}(M / Y ; E)$, which is canonically identified with $\mathcal{C}^{\infty}(M ; E)$. The choice of connections induces a connection on $\mathcal{C}^{\infty}(\phi ; E)$ through the covariant differential

$$
\nabla_{X}^{\phi, E} u=\nabla_{X_{H}}^{E} \tilde{u}, \mathcal{C}^{\infty}(M / Y ; E) \ni u=\tilde{u} \in \mathcal{C}^{\infty}(M ; E),
$$

where $X_{H}$ is the horizontal lift of $X \in \mathcal{C}^{\infty}(Y ; T Y)$. The curvature of this connection is a 2 -form on the base with values in the first-order differential operators on sections of $E$ on the fibres

$$
\omega=\left(\nabla^{\phi, E}\right)^{2} \in \Lambda^{2} Y \otimes_{\mathcal{C}^{\infty}(Y)} \operatorname{Diff}^{1}(M / Y ; E) .
$$

This covariant differential can be extended to the bundle $\Psi^{m}(\phi ; E)$, for each $m$ including $m=-\infty$, which has fibre $\Psi^{m}\left(Z_{y}, E_{y}\right)$ at $y$, and space of global smooth sections $\Psi^{m}(M / Y ; E)$ through its action on $\mathcal{C}^{\infty}(M ; E)$ :

$$
\nabla^{\phi, E} Q=\left[\nabla^{\phi, E}, Q\right], \quad Q \in \Psi^{m}(M / Y ; E) .
$$

The curvature of the induced connection is given by the commutator action of the curvature

$$
\left(\nabla^{\phi, E}\right)^{2}=[\omega, \cdot] .
$$

Let $\pi: G^{-\infty}(\phi ; E) \longrightarrow Y$ be the infinite-dimensional bundle over $Y$ with fibre

$$
G^{-\infty}\left(Z_{y} ; E_{y}\right)=\left\{\operatorname{Id}_{E_{y}}+Q ; Q \in \Psi^{-\infty}\left(Z_{y} ; E_{y}\right), \operatorname{Id}_{E_{y}}+Q \text { is invertible }\right\} .
$$

This is naturally identified with an open subbundle of $\Psi^{-\infty}(\phi ; E) \subset \Psi^{m}$ $(\phi ; E)$ and as such has an induced covariant differential. If $\sigma \in G^{-\infty}$ $(M / Y ; E)$ is a global section, the corresponding odd Chern character is

$$
\begin{array}{r}
\mathrm{Ch}_{\text {odd }}\left(\sigma, \nabla^{\phi, E}\right)=\frac{1}{2 \pi i} \operatorname{Tr}\left(\int_{0}^{1}\left(\sigma^{-1} \nabla^{\phi, E} \sigma\right) \exp \left(\frac{w\left(s, \sigma, \nabla^{\phi, E}\right)}{2 \pi i}\right) d s\right), \\
\text { where } w(s, \sigma, \nabla)=s(1-s)\left(\sigma^{-1} \nabla \sigma\right)\left(\sigma^{-1} \nabla \sigma\right)+(s-1) \omega-s \sigma^{-1} \omega \sigma .
\end{array}
$$


Even though the curvature $\omega$ from (4.4) is not of trace class, the term $\sigma^{-1} \nabla^{\phi, E} \sigma$ is a 1-form with values in smoothing operators, the identity being annihilated by the covariant differential, so the argument of $\mathrm{Tr}$ is a smoothing operator.

The form in (4.8) is the pull-back under the section $\sigma$ of a 'universal' odd Chern character on the total space of the bundle. To see this, first pull the bundle back to its own total space

$$
\pi^{*} G^{-\infty}(\phi ; E) \longrightarrow G^{-\infty}(\phi ; E)
$$

This has a tautological section

$$
a: G^{-\infty}(\phi ; E) \longrightarrow \pi^{*} G^{-\infty}(\phi ; E)
$$

and carries the pulled back covariant differential $\tilde{\nabla}^{\phi, E}=\pi^{*} \nabla^{\phi, E}$. The geometric odd Chern character on $G^{-\infty}(\phi ; E)$ is

$$
\begin{gathered}
\mathrm{Ch}_{\text {odd }}\left(\tilde{\nabla}^{\phi, E}\right)=\frac{1}{2 \pi i} \operatorname{Tr}\left(\int_{0}^{1} a^{-1} \tilde{\nabla}^{\phi, E} a \exp \left(\frac{w\left(s, a, \tilde{\nabla}^{\phi, E}\right)}{2 \pi i}\right) d s\right) \text {, where } \\
w\left(s, a, \tilde{\nabla}^{\phi, E}\right)=s(1-s)\left(a^{-1} \tilde{\nabla}^{\phi, E} a\right)\left(a^{-1} \tilde{\nabla}^{\phi, E} a\right)+(s-1) \tilde{\omega}-s a^{-1} \tilde{\omega} a
\end{gathered}
$$

here $\tilde{\omega}=\pi^{*} \omega$ is the pull-back of the curvature. This clearly has the desired universal property for smooth sections:

$$
\mathrm{Ch}_{\text {odd }}\left(\sigma, \nabla^{\phi, E}\right)=\sigma^{*} \mathrm{Ch}_{\text {odd }}\left(\tilde{\nabla}^{\phi, E}\right) \text {. }
$$

The basic properties of the geometric Chern character are well known and discussed, for example, in [1]. In particular of course, the forms are closed. This follows from identities for the forms $w=w\left(s, a, \tilde{\nabla}^{\phi, E}\right)$ and $\theta=$ $a^{-1} \tilde{\nabla}^{\phi, E} a$ in (4.11) which will be used below. Namely the Bianchi identity for the connection implies (cf. (3.5) in [1] ) that

$$
\begin{aligned}
\tilde{\nabla}^{\phi, E} w & =s[w, \theta] \text { and hence } \\
\tilde{\nabla}^{\phi, E}\left(\theta \exp \left(\frac{w}{2 \pi i}\right)\right)= & \left.-\frac{d w}{d s} \exp \left(\frac{w}{2 \pi i}\right)\right)-s\left[\theta \exp \left(\frac{w}{2 \pi i}\right), \theta\right], \\
= & -2 \pi i \frac{d}{d s} \exp \left(\frac{w}{2 \pi i}\right)+\int_{0}^{1}\left[e^{\frac{(1-r) w}{2 \pi i}}, \frac{d w}{d s} e^{\frac{r w}{2 \pi i}}\right] d r \\
& -s\left[\theta \exp \left(\frac{w}{2 \pi i}\right), \theta\right] .
\end{aligned}
$$


All the commutators have vanishing trace so

$$
\begin{aligned}
d \mathrm{Ch}_{\text {odd }} & =\frac{1}{2 \pi i} \operatorname{Tr} \tilde{\nabla}^{\phi, E}\left(\int_{0}^{1} a^{-1} \tilde{\nabla}^{\phi, E} a \exp \left(\frac{w\left(s, a, \tilde{\nabla}^{\phi, E}\right)}{2 \pi i}\right) d s\right) \\
& =-\operatorname{Tr} \int_{0}^{1} \frac{d}{d s} \exp \left(\frac{w\left(s, a, \tilde{\nabla}^{\phi, E}\right)}{2 \pi i}\right) d s=0
\end{aligned}
$$

since $\operatorname{Tr}\left(e^{\frac{w(0)}{2 \pi i}}-e^{\frac{w(1)}{2 \pi i}}\right)=0$.

LEMMA 2. Under the inversion map $I: G^{-\infty}(\phi, E) \ni \sigma \longmapsto \sigma^{-1} \in G^{-\infty}$ $(\phi ; E)$ the Chern character pulls back to its negative $I^{*} \mathrm{Ch}_{\text {odd }}=-\mathrm{Ch}_{\text {odd }}$.

Proof. This follows directly from (4.8) since

$$
w\left(s, \sigma^{-1}, \nabla\right)=\sigma w(1-s, \sigma, \nabla) \sigma^{-1}, \quad \sigma \nabla^{\phi, E} \sigma^{-1}=-\left(\nabla^{\phi, E} \sigma\right) \sigma^{-1}
$$

and the conjugation invariance of the trace.

Furthermore, the cohomology class defined by $\mathrm{Ch}_{\text {odd }}$ is additive, in the sense that

$$
\mathrm{Ch}_{\text {odd }}\left(\sigma_{1} \sigma_{2}\right)=\mathrm{Ch}_{\text {odd }}\left(\sigma_{1}\right)+\mathrm{Ch}_{\text {odd }}\left(\sigma_{2}\right)+d F \text {. }
$$

Again it is useful to give a universal version of such a multiplicativity formula.

Let $\left(G^{-\infty}(\phi, E)\right)^{[2]}$ be the fibre product of $G^{-\infty}(\phi, E)$ with itself as a bundle over $Y$. Then there are the usual three maps

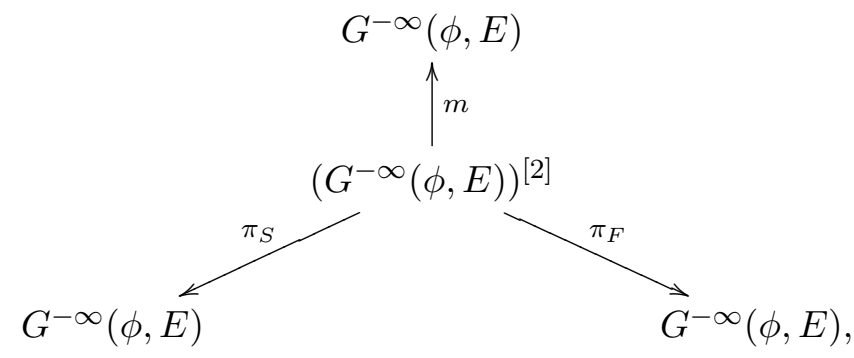

where $\pi_{F}(a, b)=b, \pi_{S}(a, b)=a$ and $m(a, b)=a b$.

Proposition 3. There is a smooth form $\mu$ on $\left(G^{-\infty}(\phi, E)\right)^{[2]}$ such that

$$
m^{*} \mathrm{Ch}_{o d d}=\pi_{F}^{*} \mathrm{Ch}_{o d d}+\pi_{S}^{*} \mathrm{Ch}_{o d d}+d \mu .
$$

Proof. This follows from essentially the same argument as used in the proof of Proposition 1. Thus, consider the bundle of groups with $E$ replaced by $E \oplus E$ in which the original is embedded as acting on the first copy and as the identity on the second copy. As in (2.15), this action on $E \oplus E$ is homotopic under a family of $2 \times 2$ absolute rotations, i.e. not depending on the space variables, to the action on the second copy with the identity 
on the first. Now, $m^{*} \mathrm{Ch}_{\text {odd }}$ is realized through the product, i.e. diagonal action on the first factor. Applying the rotations but just in the second term of this product action, the map $m$ is homotopic to $\pi_{S} \oplus \pi_{F}$. Since the Chern character is closed, the same argument as in Proposition 1 constructs the transgression form $\mu$ as the integral of the variation along the homotopy.

\section{Odd elliptic families}

Now we turn to the consideration of a given family of self-adjoint elliptic pseudodifferential operators, $A \in \Psi^{1}(M / Y ; E)$. In fact it is not selfadjointness that we need here, but rather the consequence that $A+i t$, which is a product-type family in the space $\Psi_{\mathrm{ps}}^{1,1}(M / Y ; E)$, should be fully elliptic and hence invertible for large real $t,|t|>T$. See $[\mathbf{2 5}]$ and $[\mathbf{2 4}]$ for a discussion of product-suspended pseudodifferential operators. More generally we may simply start with an elliptic family in this sense, possibly of different order, $A \in \Psi_{\mathrm{ps}}^{m, l}(M / Y ; E)$. It follows from the assumed full ellipticity that for each value of the parameter $y \in Y$ the set of invertible perturbations

$$
\begin{aligned}
\mathcal{A}_{y}=\left\{A+i t+q(t) ; q \in \Psi_{\text {sus }}^{-\infty}\left(Z_{y} ; E_{y}\right),\right. & \left.(A+i t+q(t))^{-1} \in \Psi^{-1}\left(Z_{y} ; E_{y}\right) \forall t \in \mathbb{R}\right\} \text { or } \\
\mathcal{A}_{y}=\left\{A(t)+q(t) ; q \in \Psi_{\text {sus }}^{-\infty}\left(Z_{y} ; E_{y}\right),\right. & \left.(A(t)+q(t))^{-1} \in \Psi^{-m}\left(Z_{y} ; E_{y}\right) \forall t \in \mathbb{R}\right\}
\end{aligned}
$$

is non-empty. This is discussed in the proof below.

Proposition 4. If $A \in \Psi^{1}(M / Y ; E)$ is an elliptic and self-adjoint family or $A \in \Psi_{\mathrm{ps}}^{m, l}(M / Y ; E)$ is a fully elliptic product-type family then (5.1) defines a smooth (infinite dimensional) Fréchet subbundle $\mathcal{A}(\phi) \subset \Psi_{\mathrm{ps}}^{m, l}(\phi, E)$ (where $m=l=1$ in the standard case) over $Y$ with fibres which are principal spaces for the action of the bundle of groups $G_{\text {sus }}^{-\infty}(\phi ; E)$.

Proof. The non-emptiness of the fibre at any point follows from standard results for the even index. Namely at each point in the base, the family $A_{y}(t)$ is elliptic and invertible for large $|t|$ as a consequence of the assumed full ellipticity. Thus the index of this family is an element of $K_{c}^{0}(\mathbb{R})$ and hence vanishes. For such a family there is a compactly supported, in the parameter, family $q(t)$ of smoothing operators on the fibre which is such that $A(t)+q(t)$ is invertible for all $t \in \mathbb{R}$.

Now the fact that the fibre is a principal space for the group $G_{\text {sus }}^{-\infty}\left(Z_{y} ; E_{y}\right)$ follows directly, since for two such perturbations $q_{i}, i=1,2$,

$$
A(t)+q_{1}(t)=\left(\operatorname{Id}_{E_{y}}+q_{12}(t)\right)\left(A(t)+q_{2}(t)\right), \quad q_{12} \in \Psi_{\text {sus }}^{-\infty}\left(Z_{y} ; E_{y}\right)
$$

and conversely. The local triviality of this bundle follows from the fact that invertibility persists under small perturbations. 
It is this bundle, $\mathcal{A}(\phi)$, which we think of as the index bundle since the existence of a global section is equivalent to the vanishing, in odd K-theory, of the index of the original family. Note that since we permit the orders $m$ and $l$ of a fully elliptic family in $\Psi_{\mathrm{ps}}^{m, l}(\phi, E)$ to take values other than 1 , the index is additive in the sense that the index bundles of two elliptic families (acting on the same bundle) compose in the obvious way.

\section{Eta forms for an odd family}

To define the geometric eta form, recall that it is shown above that a covariant derivative is induced on the bundle $\Psi^{m}(\phi ; E) \longrightarrow Y$ from the connection on $\phi$ and the connection on $E$. Consider the subbundle of elliptic and invertible pseudodifferential operators, $G^{m}(\phi ; E)$. Since product-suspended operators can be seen as one-parameter families of pseudodifferential operators, there is an evaluation map

$$
\mathrm{ev}: \mathbb{R}_{\tau} \times \mathcal{A}(\phi) \ni(\tau, a) \longmapsto a(\tau) \in G^{m}(\phi ; E)
$$

compatible with the bundle structure. On $G^{m}(\phi ; E)$, consider as before the tautological bundle

$$
\pi^{*} G^{m}(\phi ; E) \longrightarrow G^{m}(\phi ; E)
$$

obtained by pulling back the bundle to its own total space. With $a: G^{m}$ $(\phi ; E) \longrightarrow \pi^{*} G^{m}(\phi ; E)$ the tautological section consider the odd form

$$
\lambda=\frac{1}{2 \pi i} a^{-1} \tilde{\nabla} a \int_{0}^{1} \exp \left(\frac{s(1-s)\left(a^{-1} \tilde{\nabla} a\right)\left(a^{-1} \tilde{\nabla} a\right)+(s-1) \tilde{\omega}-s a^{-1} \tilde{\omega} a}{2 \pi i}\right) d s
$$

taking values in $\pi^{*} \Psi^{0}(\phi ; E)$. Here $\tilde{\nabla}=\pi^{*} \nabla$ and $\tilde{\omega}=\pi^{*} \omega$ with $\omega$ defined in (4.4).

This is formally the same as the argument of the trace functional in (4.11), except of course that now the section $a$ is no longer a perturbation of the identity by a smoothing operator, but an invertible operator of order $m$. Nevertheless, the identities in (4.13) still hold, since they are based on the Bianchi identity. Writing the pull-back under the evaluation map as

$$
\operatorname{ev}^{*}(\lambda)=\lambda^{t}+\lambda^{n} \wedge d \tau, \quad \lambda^{n}=\iota_{\partial \tau} \lambda
$$

both the tangential and normal parts are form-valued sections of the bundle

$$
\pi_{\mathcal{A}}^{*} \Psi_{\mathrm{ps}}^{0,0}(\phi ; E) \longrightarrow \mathcal{A}
$$

obtained by pulling back $\pi: \Psi_{\mathrm{ps}}^{0,0}(\phi ; E) \longrightarrow Y$ to $\mathcal{A}$.

Definition 2. On the total space of the bundle $\mathcal{A}$ the (even) geometric eta form is

$$
\eta_{\mathcal{A}}=\operatorname{Tr}_{\mathrm{sus}}\left(\lambda^{n}\right)
$$


where $\operatorname{Tr}_{\text {sus }}$ is the regularized trace of [20] taken fibrewise in the fibres of the bundle (6.5).

The bundle (6.5) does not have a tautological section, but

$$
\pi_{\mathcal{A}}^{*} G_{\mathrm{ps}}^{m, l}(\phi ; E) \longrightarrow \mathcal{A}
$$

does, where $G_{\mathrm{ps}}^{m, l}(\phi ; E) \subset \Psi_{\mathrm{ps}}^{m, l}(\phi ; E)$ is the subbundle of elliptic invertible elements, with product-type pseudodifferential inverses. Denote this section by $\alpha_{\mathcal{A}}: \mathcal{A} \longrightarrow \pi_{\mathcal{A}}^{*} G_{\mathrm{ps}}^{m, l}(\phi ; E)$. Then consider also the odd form

$$
\begin{aligned}
\tilde{\gamma}_{\mathcal{A}}= & \frac{1}{2 \pi i} \widetilde{\operatorname{Tr}}\left[\int_{0}^{1} \alpha_{\mathcal{A}}^{-1} \hat{\nabla} \alpha_{\mathcal{A}}\right. \\
& \left.\times \exp \left(\frac{s(1-s)\left(\alpha_{\mathcal{A}}^{-1} \hat{\nabla} \alpha_{\mathcal{A}}\right)^{2}+(s-1) \hat{\omega}-s \alpha_{\mathcal{A}}^{-1} \hat{\omega} \alpha_{\mathcal{A}}}{2 \pi i}\right) d s\right]
\end{aligned}
$$

where $\widetilde{T r}$ is the formal trace from $[\mathbf{2 0}]$ and $\hat{\nabla}=\pi_{\mathcal{A}}^{*} \nabla, \hat{\omega}=\pi_{\mathcal{A}}^{*} \omega$.

Proposition 5. For an odd elliptic family of first order (so either selfadjoint or directly of product type), the exterior derivative of the geometric eta form

$$
d \eta_{\mathcal{A}}=\tilde{\gamma}_{\mathcal{A}}=\pi_{\mathcal{A}}^{*} \gamma_{\mathcal{A}}
$$

is the pull-back of a closed form on the base $\gamma_{A} \in \mathcal{C}^{\infty}\left(Y ; \Lambda^{\text {odd }}\right)$.

PROOF. Recall first that the regularized trace is defined in [20] by taking the constant term in the asymptotic expansion of

$$
\int_{-\tau}^{\tau} \int_{0}^{\tau_{p}} \cdots \int_{0}^{\tau_{1}} \operatorname{Tr}\left(\frac{\partial^{p}}{\partial r^{p}} \lambda^{t}(r)\right) d r d \tau_{1} \cdots d \tau_{p}
$$

as $\tau \rightarrow+\infty$. Here $p \in \mathbb{N}$ is chosen large enough so that $\frac{\partial^{p}}{\partial \tau^{p}} \lambda^{t}(\tau)$ is of trace class - the product-suspended property implies that high $\tau$ derivatives are of correspondingly low order in both senses. This is a trace, i.e. vanishes on commutators, but is not exact in the sense that $\widetilde{\operatorname{Tr}}(A)=\operatorname{Tr}_{\text {sus }}\left(\frac{\partial A}{\partial \tau}\right)$ does not necessarily vanish, but is determined by the asymptotic expansions of $A(\tau)$ as $\pm \tau \rightarrow \infty$ since it does vanish for smoothing Schwartz perturbations of $A$.

As noted above, the identities (4.13) hold for $\lambda$. For the pull-back under the evaluation map this means that modulo commutators

$$
\tilde{\nabla} \lambda^{n} \equiv \frac{\partial}{\partial \tau} \lambda^{t}
$$

Now, taking $p$ further derivatives with respect to $\tau$ gives the same identity modulo commutators where the sum of the orders of the terms becomes low 
as $p$ increase. So, applying the trace functional the commutator terms vanish and it follows that

$$
\operatorname{Tr}\left(\frac{\partial^{p}}{\partial \tau^{p}} \tilde{\nabla} \lambda^{n}(\tau)\right) \wedge d \tau=\operatorname{Tr}\left(\frac{\partial^{p}}{\partial \tau^{p}} \frac{\partial}{\partial \tau} \lambda^{t}(r)\right) \wedge d \tau .
$$

Therefore,

$$
\begin{aligned}
d \eta_{\mathcal{A}} & =\operatorname{Tr}_{\text {sus }}\left(\tilde{\nabla} \lambda^{n}\right)=\operatorname{Tr}_{\text {sus }}\left(\frac{\partial}{\partial \tau} \lambda^{t}\right) \\
& =\widetilde{\operatorname{Tr}}\left(\lambda^{t}\right)=\tilde{\gamma}_{\mathcal{A}}
\end{aligned}
$$

by definition of the formal trace.

As already noted, the formal trace vanishes on low order perturbations so $\tilde{\gamma}_{\mathcal{A}}$ is basic, i.e. is actually the pull-back of a well-defined form $\gamma_{A}$ on $Y$ depending only on the initial family $A$.

The index formula (4) therefore amounts to showing that the form $\gamma_{A}$ represents the (odd) Chern character of the index of the family $A$ in cohomology. This is difficult to approach directly, computationally, so instead we show how the index bundle $\mathcal{A}(\phi)$ can be 'trivialized' by extending the bundle of structure groups.

If $A \in \Psi_{\mathrm{ps}}^{m, l}(M / Y ; E)$ is a fully elliptic family then it has an 'inverse' family which, whilst not completely well-defined, is determined up to smoothing terms. Namely the bundle $\mathcal{A}$ is locally trivial over $Y$ and in particular has local sections. Taking a partition of unity $\psi_{i}$ on $Y$ subordinate to a cover by open set $U_{i}$ over each of which there is a section $A_{i}$ the inverse family can be taken to be

$$
\sum_{i} \psi_{i}(y) A_{i}^{-1} \in \Psi_{\mathrm{ps}}^{-m,-l}(M / Y ; E)
$$

It is fully elliptic and, essentially by definition, the corresponding bundle of invertible perturbations is naturally identified with the bundle $\mathcal{A}^{-1}(\phi) \subset$ $\Psi_{\mathrm{ps}}^{-m,-l}(\phi ; E)$ consisting of the inverses of the elements of $\mathcal{A}(\phi)$.

Lemma 3. Under the inversion map $\mathcal{A}(\phi) \longrightarrow \mathcal{A}^{-1}(\phi)$ the eta form $\eta_{\mathcal{A}^{-1}}$ on $\mathcal{A}^{-1}(\phi)$ associated with the inverse family (6.14) pulls back to $-\eta_{\mathcal{A}}$.

Proof. The proof is similar to the one of Lemma 2, namely since the regularized trace vanishes on commutators, the result follows from the analog of (4.15) for the bundles $\mathcal{A}(\phi)$ and $\mathcal{A}^{-1}(\phi)$.

We also need a variant of the multiplicative formula (4.18). For this, consider the fibre product $\mathcal{A}^{[2]}(\phi)$ of two copies of the bundle $\mathcal{A}(\phi)$ and the product map given by inversion in the second map

$$
\tilde{m}: \mathcal{A}^{[2]}(\phi) \ni\left(A^{\prime}, A\right) \longmapsto A^{\prime} A^{-1} \in G_{\text {sus }}^{-\infty}(\phi ; E) .
$$


Proposition 6. Under pull-back under the three maps

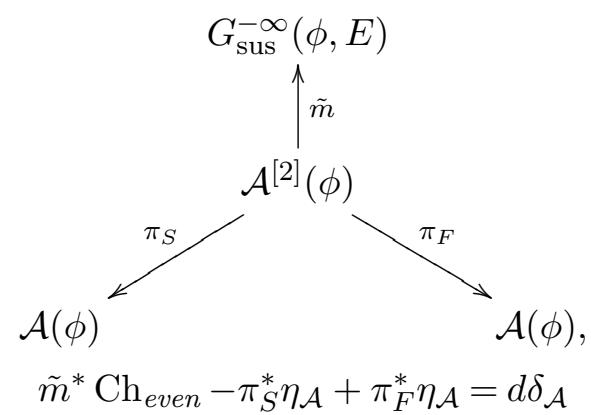

for a smooth form $\delta_{\mathcal{A}}$ on $\mathcal{A}^{[2]}(\phi)$.

Proof. We perform the same deformation as in Proposition 3 and its earlier variants. Taking into account Proposition 5 and Lemma 2, it follows that the same conclusion holds except that extra terms may appear from $\tau \rightarrow \pm \infty$. These give a basic form so in place of the desired identity (6.17) we find instead that

$$
\tilde{m}^{*} \mathrm{Ch}_{\mathrm{even}}-\pi_{S}^{*} \eta_{\mathcal{A}}+\pi_{F}^{*} \eta_{\mathcal{A}}=d \delta_{\mathcal{A}}^{\prime}+\pi^{*} \mu
$$

where $\delta_{\mathcal{A}}^{\prime}$ is smooth form on $\mathcal{A}^{[2]}(\phi)$ and $\mu$ is smooth form on the base, with $\pi: \mathcal{A}^{[2]}(\phi) \longrightarrow Y$. However, under exchange of the two factors, the left side of (6.18) changes sign, while the final, basic, term is unchanged. Thus taking the odd part of (6.18) gives (6.17).

It is also of interest to see how the eta form transforms under a change of connections for the fibration $\phi: M \rightarrow Y$ and the bundle $E \rightarrow M$.

Lemma 4. If $\eta_{\mathcal{A}}$ and $\eta_{\mathcal{A}}^{\prime}$ are eta forms associated to the self-adjoint elliptic family $A \in \Psi^{1}(M / Y ; E)$ with respect to two different choices of connections for the fibration $\phi: M \rightarrow Y$ and the vector bundle $E \rightarrow M$, then there exist a form $\beta \in \Omega^{\text {even }}(Y)$ and a form $\alpha \in \Omega^{\text {odd }}(\mathcal{A})$ such that

$$
\eta_{\mathcal{A}}^{\prime}-\eta_{\mathcal{A}}=\pi_{\mathcal{A}}^{*} \beta+d \alpha .
$$

Proof. Consider the new fibration $\phi \times \mathrm{Id}: M \times[0,1]_{t} \rightarrow Y \times[0,1]_{t}$ with family $\operatorname{pr}^{*} A$ where $\operatorname{pr}: M \times[0,1] \rightarrow M$ is the projection on the left factor. Clearly, we can choose connections for the fibration $\phi \times$ Id and the bundle $\operatorname{pr}^{*} E \rightarrow M \times[0,1]_{t}$ such that if $\eta_{\mathrm{pr}^{*} \mathcal{A}}$ is the corresponding eta form, then

$$
\iota_{0}^{*} \eta_{\mathrm{pr}^{*} \mathcal{A}}=\eta_{\mathcal{A}}, \quad \iota_{1}^{*} \eta_{\mathrm{pr}^{*} \mathcal{A}}=\eta_{\mathcal{A}}^{\prime},
$$

where for $i \in[0,1], \iota_{i}: \mathcal{A} \rightarrow \mathcal{A} \times\{i\} \subset \mathcal{A} \times[0,1]_{t} \cong \mathrm{pr}^{*} \mathcal{A}$ is the natural inclusion. The form $\eta_{\mathrm{pr}^{*} \mathcal{A}}$ can be written as

$$
\eta_{\mathrm{pr}^{*} \mathcal{A}}=\operatorname{pr}_{\mathcal{A}}^{*} \omega_{0}(t)+\operatorname{pr}_{\mathcal{A}}^{*} \omega_{1}(t) \wedge d t, \quad \omega_{0}(t) \in \Omega^{\text {even }}(\mathcal{A}), \omega_{1}(t) \in \Omega^{\text {odd }}(\mathcal{A}),
$$


where $\operatorname{pr}_{\mathcal{A}}: \operatorname{pr}^{*} \mathcal{A} \cong \mathcal{A} \times[0,1] \rightarrow \mathcal{A}$ is the natural projection. This suggests to define $\alpha \in \Omega^{\text {even }}(\mathcal{A})$ by

$$
\alpha=\left(\operatorname{pr}_{\mathcal{A}}\right)_{*}\left(\eta_{\operatorname{pr}^{*} \mathcal{A}}\right)=\int_{0}^{1} \omega_{1}(t) d t .
$$

Then we have that

$$
\begin{aligned}
d \alpha & =\left(\operatorname{pr}_{\mathcal{A}}\right)_{*}\left(d \eta_{\mathrm{pr}^{*} \mathcal{A}}\right)+\eta_{\mathcal{A}}^{\prime}-\eta_{\mathcal{A}} \\
& =\pi_{\mathcal{A}}^{*} \operatorname{pr}_{*}\left(\gamma_{\mathrm{pr}^{*} \mathcal{A}}\right)+\eta_{\mathcal{A}}^{\prime}-\eta_{\mathcal{A}}
\end{aligned}
$$

Thus, the result follows by taking $\beta=-\operatorname{pr}_{*}\left(\gamma_{\mathrm{pr}^{*} \mathcal{A}}\right)=-\int_{0}^{1} v_{1}(t) d t$ where

$$
\gamma_{\mathrm{pr}^{*} \mathcal{A}}=\operatorname{pr}^{*} v_{0}(t)+\operatorname{pr}^{*} v_{1}(t) \wedge d t, \quad v_{0}(t) \in \Omega^{\text {odd }}(Y), v_{1}(t) \in \Omega^{\mathrm{even}}(Y),
$$

and where pr also denotes the natural projection pr: $Y \times[0,1]_{t} \rightarrow Y$.

\section{Extended eta invariant}

The bundle $\mathcal{A}(\phi)$ in (5.1) is a bundle of principal spaces for the action of the fibres of $G_{\text {sus }}^{-\infty}(\phi ; E)$. The fibres can be enlarged to give an action of the central, contractible, group in (1.7) by setting

$$
\widetilde{\mathcal{A}}_{y}=\tilde{G}^{-\infty}\left(Z_{y} ; E\right) \cdot \mathcal{A}_{y} .
$$

This is not so easily characterized additively but is the image of the quotient map on the fibre product

$$
p_{\sim}: \widetilde{G}_{\text {sus }}^{-\infty}(\phi ; E) \times_{Y} \mathcal{A}(\phi) \longrightarrow \widetilde{\mathcal{A}}(\phi), \quad p_{\sim}(\tilde{g}, A)=\tilde{g} A .
$$

In particular there is an exact and fibrewise delooping sequence coming from (1.7):

$$
\mathcal{A}(\phi) \longrightarrow \widetilde{\mathcal{A}}(\phi) \stackrel{\tilde{R}_{\infty}}{\longrightarrow} \widetilde{G}_{\text {sus }}^{-\infty}(\phi ; E) .
$$

The quotient map here can be defined in the fibre $\widetilde{\mathcal{A}}_{y}$ by

$$
\tilde{R}_{\infty}\left(A_{y}^{\prime}\right)=\lim _{\tau \rightarrow \infty} A_{y}^{\prime} A_{y}^{-1}
$$

where $A_{y} \in \mathcal{A}_{y}$ is any point in the fibre of $\mathcal{A}(\phi)$ over the same basepoint. Clearly the result does not depend on this choice of $A_{y}$.

The construction above of the eta form on $\mathcal{A}(\phi)$ extends to $\widetilde{\mathcal{A}}(\phi)$. Thus, the same form (6.3) pulls back under the evaluation map

$$
\widetilde{\mathrm{ev}}: \mathbb{R}_{\tau} \times \widetilde{\mathcal{A}}(\phi) \longrightarrow G^{m}(\phi ; E)
$$

to give

$$
\widetilde{\mathrm{ev}}^{*}(\lambda)=\widetilde{\lambda}^{t}+\widetilde{\lambda}^{n} \wedge d \tau
$$

Then, extending Definition 2, set

$$
\eta_{\widetilde{\mathcal{A}}}=\operatorname{Tr}_{\mathrm{sus}}\left(\widetilde{\lambda}^{n}\right)
$$


We use this extended bundle and eta form to analyse the invariance properties of $\eta_{\mathcal{A}}$.

Consider the fibre product with projections and quotient map

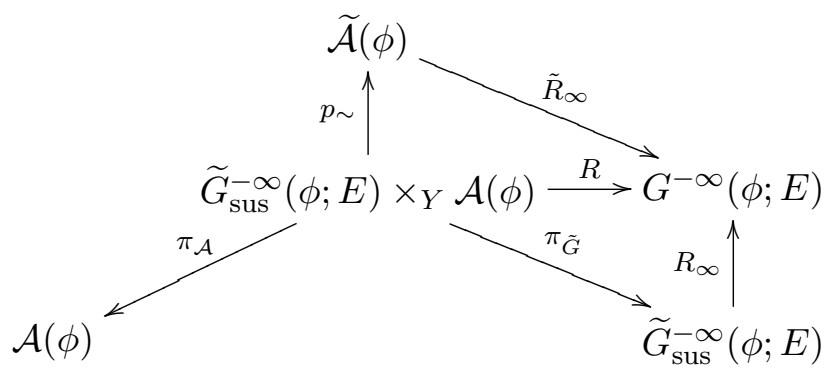

Proposition 7. The diagram (7.8) commutes and there are smooth forms $\tilde{\delta}_{\mathcal{A}}$ and $\tilde{\mu}_{\mathcal{A}}$ respectively on the fibre product and $G^{-\infty}(\phi ; E)$ such that the three eta forms pull back to satisfy

$$
p_{\sim}^{*} \eta_{\widetilde{\mathcal{A}}}=\pi_{\mathcal{A}}^{*} \eta_{\mathcal{A}}+\pi_{\tilde{G}}^{*} \tilde{\eta}+d \tilde{\delta}_{A}+R^{*} \tilde{\mu}_{\mathcal{A}}
$$

Proof. The commutativity of the parallelogram on the right is discussed above and defines the diagonal map, $R$.

The formula (7.9) is a generalization of that of Proposition 3 and the proof proceeds along the same lines. Consider the odd Chern character on $E \oplus E$. Thus, from the fibre product there are two evaluation maps, $\widetilde{\text { Ev }}$ and $\widetilde{\mathrm{ev}}$ and we may combine these using the bundle rotation as in (2.13). This gives the two-parameter family of maps from $\widetilde{G}_{\text {sus }}^{-\infty}(\phi ; E) \times_{Y} \mathcal{A}(\phi)$ :

$$
\begin{aligned}
& {[0,1]_{t} \times \mathbb{R} \times \widetilde{G}_{\text {sus }}^{-\infty}(\phi ; E) \times_{Y} \mathcal{A}(\phi) \ni(t, \tau, \tilde{g}, \widetilde{A})} \\
& \quad \longmapsto M^{-1}(t)\left(\begin{array}{cc}
\tilde{g}_{y}(\tau) & 0 \\
0 & \text { Id }
\end{array}\right) M(t)\left(\begin{array}{cc}
\text { Id } & 0 \\
0 & \widetilde{A}_{y}(\tau)
\end{array}\right) \in G^{m}(\phi, E \oplus E) .
\end{aligned}
$$

Pulling back the form $\lambda$ of (6.3) under this map and 'integrating' over $[0,1]_{t} \times \mathbb{R}_{\tau}$ gives the identity (7.9), where the $\tau$ integral is to be interpreted as part of the regularized trace. Since the form $\lambda$ is closed modulo commutators, if the product decomposition of its pull-back is

$$
d t \wedge d \tau \wedge \mu+d t \wedge \lambda_{t}+d \tau \wedge \lambda_{\tau}+\lambda^{\prime}
$$

then

$$
d \mu-\frac{\partial \lambda_{t}}{\partial \tau}+\frac{\partial \lambda_{\tau}}{\partial t} \equiv 0
$$

again modulo commutators. The regularized trace and integral of the last term gives the difference of the three pulled-back eta forms and $\mu$ defines the term $\tilde{\delta}_{\mathcal{A}}$ on the fibre product.

Thus it remains to analyse the second term in (7.12). The exterior differentials in (4.11) each fall on either a factor from $\tilde{G}_{\text {sus }}^{-\infty}(\phi ; E)$ or on $\mathcal{A}$. The terms involving no derivative of the first type, so the 'pure $\mathcal{A}$ part', makes no 
contribution, since as discussed earlier, the rotation factor $M(t)$ disappears. Thus, only terms with at least one derivative falling on the first three factors of (7.10) need to be considered. This results in a smoothing operator and the regularization of the trace functional is not necessary. Then the $\tau$ integral reduces to the value of $\lambda_{t}$ at $\tau=\infty$ which depends only on the leading term in $\mathcal{A}$ as $\tau \rightarrow \infty$, which is to say the corresponding term in $A$ itself, and $R_{\infty}(\tilde{g})$. This leads to the additional term $\tilde{\mu}_{A}$ in (7.9).

The left action of the groups on the fibres of the index bundle induces a contraction map

$$
\begin{gathered}
L: G_{\text {sus }}^{-\infty}(\phi ; E) \times_{Y} \mathcal{A}(\phi) \longrightarrow \mathcal{A}(\phi) \\
L(s, u)=s u, \quad \forall s \in G^{-\infty}\left(Z_{y} ; E_{y}\right), \quad u \in \mathcal{A}_{y} \quad \forall y \in Y .
\end{gathered}
$$

Corollary 1. There is a smooth odd form $\tilde{\delta}$ on $G_{\mathrm{sus}}^{-\infty}(\phi ; E) \times_{Y} \mathcal{A}(\phi)$ such that

$$
L^{*} \eta_{\mathcal{A}}=\pi_{\mathcal{A}}^{*} \eta_{\mathcal{A}}+\pi_{G}^{*} \mathrm{Ch}_{\text {even }}+d \tilde{\delta} .
$$

Proof. Restricting to the subbundle $G_{\text {sus }}^{-\infty}(\phi ; E)$ in $(7.8)$ gives a diagram which includes into it and on which $R_{\infty}$ and $R$ are trivial:

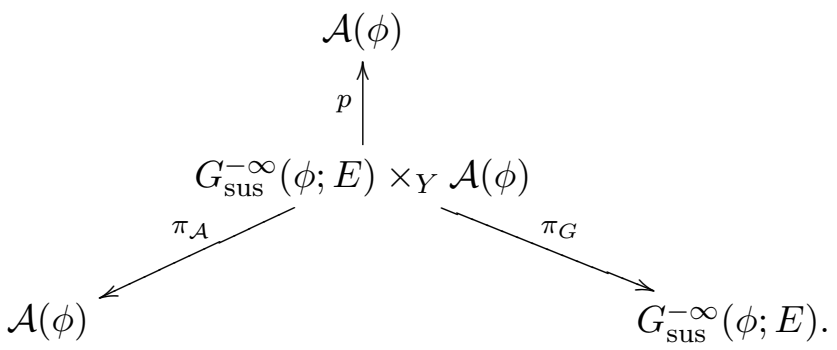

Thus (7.9) restricts to this diagram with $\tilde{\mu}_{A}$ vanishing and $\tilde{\eta}$ reducing to $\mathrm{Ch}_{\text {even }}$ on $G_{\text {sus }}^{-\infty}(\phi ; E)$. Thus (7.14) follows.

REMARK 1. If $s: U \longrightarrow G_{\text {sus }}^{-\infty}(\phi ; E)$ and $\alpha: U \longrightarrow \mathcal{A}$ are sections over an open subset $U \subset Y$, then corollary 1 shows that

$$
\eta(s \alpha)-\eta(\alpha)=\mathrm{Ch}_{\text {even }}(s)+d \alpha^{*} \tilde{\delta} .
$$

\section{Index formula: Proof of Theorem 1}

The bundle $\widetilde{\mathcal{A}}(\phi)$ has contractible fibres and hence has a global continuous section $\tilde{A}: Y \longrightarrow \widetilde{\mathcal{A}}(\phi)$; this section is easily made smooth. The inverse image of the range of this section under the vertical map, $p_{\sim}$, in (7.8) is a submanifold $\mathcal{F} \subset \widetilde{G}_{\text {sus }}^{-\infty}(\phi ; E) \times_{Y} \mathcal{A}(\phi)$. Indeed for each $y \in Y$ and each $B_{y} \in \mathcal{A}_{y}$ there is a unique $Q_{y} \in \widetilde{G}_{\text {sus }}^{-\infty}\left(Z_{y} ; E\right)$ such that

$$
Q(\tau) B_{y}=\tilde{A}_{y}(\tau)
$$


is the value of the section at that point. Thus, $\pi_{\mathcal{A}}$ restricts to an isomorphism from $\mathcal{F}$ to $\mathcal{A}$.

Using the section $\tilde{\mathcal{A}}: Y \longrightarrow \tilde{\mathcal{A}}(\phi)$, we can identify $p_{\sim}(\mathcal{F})$ with $Y$ so that restricting (7.8) to $\mathcal{F}$ gives the commutative diagram

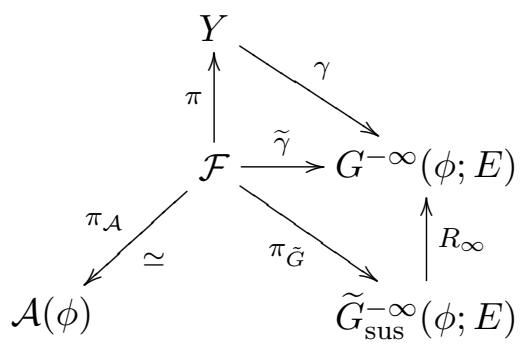

where $\widetilde{\gamma}$ is the restriction of $R$ to $\mathcal{F}$ and $\gamma$ is the classifying map defined to make this diagram commutes.

Restricted to $\mathcal{F}$ the identity (7.9) becomes modulo exact forms

$$
\pi_{\mathcal{A}}^{*} \eta_{\mathcal{A}}+\pi_{\tilde{G}}^{*} \tilde{\eta}=\pi^{*} \beta_{\tilde{A}}, \quad \beta_{\tilde{A}}=\tilde{A}^{*} \widetilde{\eta}_{\tilde{A}}-\gamma^{*} \tilde{\mu}_{\mathcal{A}} \in \mathcal{C}^{\infty}\left(Y ; \Lambda^{\text {even }}\right) .
$$

From (3.6)

$$
\pi_{\tilde{G}}^{*} d \tilde{\eta}=\widetilde{\gamma}^{*}\left(\mathrm{Ch}_{\text {odd }}\right)
$$

so pulling back to $\mathcal{A}$ under the isomorphism $\pi_{\mathcal{A}}$ gives the index formula (4):

$$
d \eta_{\mathcal{A}}=-\pi^{*} \gamma^{*}\left(\mathrm{Ch}_{\text {odd }}\right)+d \beta_{\tilde{A}} .
$$

Since the homotopy class of the section $\gamma$ represents minus the index class, this shows that $\gamma_{A}$ in (6.9) represents the Chern character of the index.

\section{Determinant of an odd elliptic family}

The eta invariant, interpreted here as the degree zero part in the eta form (there is a factor of 2 compared to the original normalization of Atiyah, Patodi and Singer) is a normalized log-determinant. In the universal case, for the classifying spaces, $\tilde{\eta}^{0}$ is a well-defined function on $\tilde{G}_{\text {sus }}^{-\infty}(Z ; E)$ and then

$$
\operatorname{det}(g)=\exp \left(2 \pi i \tilde{\eta}^{0}\right) \text { is the Freholm determinant on } G^{-\infty}(Z ; E) .
$$

In the geometric case essentially the same result is true.

Proposition 8. For $A \in \Psi_{\mathrm{ps}}^{m, k}(M / Y ; E)$ a fully elliptic family of product-type operators on the fibres of a fibration,

$$
\tau(A)=\exp \left(2 \pi i \eta_{\mathcal{A}}^{0}\right) \in \mathcal{C}^{\infty}\left(Y ; \mathbb{C}^{*}\right),
$$

where $\eta_{\mathcal{A}}^{0}$ is the degree zero part in (6.6), is a multiplicative function on fully elliptic operators on a fixed bundle,

$$
\tau(A B)=\tau(A) \tau(B), \quad A \in \Psi_{\mathrm{ps}}^{m, k}(M / Y ; E), \quad B \in \Psi_{\mathrm{ps}}^{m^{\prime}, k^{\prime}}(M / Y ; E)
$$


which is constant under smoothing perturbation and which represents the class associated to ind $(A) \in \mathrm{K}^{1}(B)$ in $\mathrm{H}^{1}(Y ; \mathbb{Z})$.

Proof. Theorem 1 shows that $d \eta_{\mathcal{A}}^{0}$ defined in principle on $\mathcal{A}$, the bundle of invertible perturbations of a given fully elliptic family $A$, is basic and represents the first odd Chern class of the index. For the zero form part, (7.14) implies true multiplicativity under the action of $G_{\text {sus }}^{-\infty}(\phi ; E)$, with the zero form part of $\mathrm{Ch}_{\text {even }}$ being the numerical index. Thus indeed the tau invariant in (9.2) is a well defined function on the base which represents the first odd Chern class in integral cohomology.

Full multiplicativity follows as in [20].

\section{Doubly suspended determinant}

Let $G_{\mathrm{sus}(2)}^{-\infty}(Z ; E)$ be the double flat-smooth loop group. Thus, its elements are Schwartz functions $a: \mathbb{R}^{2} \longrightarrow \Psi^{-\infty}(Z ; E)$ such that $\operatorname{Id}+a(t, \tau)$ is invertible for each $(t, \tau) \in \mathbb{R}^{2}$. Let

$$
G_{\operatorname{sus}(2)}^{-\infty}(Z ; E)\left[\epsilon / \epsilon^{2}\right]=G_{\operatorname{sus}(2)}^{-\infty}(Z ; E) \oplus \mathcal{S}\left(\mathbb{R}^{2} ; \Psi^{-\infty}(Z ; E)\right)
$$

be the group with the truncated $*$ (or Moyal) product obtained as in [25] by adiabatic limit from the isotropic product on $\mathcal{S}\left(\mathbb{R}^{2} ; \Psi^{-\infty}(Z ; E)\right)$ and then passing to the quotient by terms of order $\epsilon^{2}$. Explicitly this product is

$$
\left(a_{0}+\epsilon a_{1}\right)[*]\left(b_{0}+\epsilon b_{1}\right)=a_{0} b_{0}+\epsilon\left(a_{0} b_{1}+a_{1} b_{0}-\frac{1}{2 i}\left(\frac{\partial a_{0}}{\partial t} \frac{\partial b_{0}}{\partial \tau}-\frac{\partial a_{0}}{\partial \tau} \frac{\partial b_{0}}{\partial t}\right)\right)
$$

where the underlying product is in $\Psi^{-\infty}(Z ; E)$.

As shown in [25], in the adiabatic limit the Fredholm determinant, for operators on $Z \times \mathbb{R}$, induces the 'adiabatic determinant'

$$
\operatorname{det}_{\mathrm{ad}}: G_{\mathrm{sus}(2)}^{-\infty}(Z ; E)\left[\epsilon / \epsilon^{2}\right] \longrightarrow \mathbb{C}^{*}, \quad \operatorname{det}_{\mathrm{ad}}\left(g_{1} g_{2}\right)=\operatorname{det}_{\mathrm{ad}}\left(g_{1}\right) \operatorname{det}_{\mathrm{ad}}\left(g_{2}\right),
$$

which, as in the unsuspended case, generates the 1-dimensional integral cohomology of $G_{\text {sus }(2)}^{-\infty}(Z ; E)\left[\epsilon / \epsilon^{2}\right]$ - which is classifying for odd K-theory. Note that there is no such multliplicative function on the leading group, without the first order (in $\epsilon$ ) 'correction' terms in (10.2).

To define the adiabatic determinant, one needs to consider the adiabatic trace on $\Psi_{\operatorname{sus}(2)}^{-\infty}(Z ; E)\left[\epsilon / \epsilon^{2}\right]$ defined by

$$
\operatorname{Tr}_{\mathrm{ad}}(a)=\frac{1}{2 \pi} \int_{\mathbb{R}^{2}} \operatorname{Tr}_{Z}\left(a_{1}(t, \tau)\right) d t d \tau, \quad a=a_{0}+\epsilon a_{1} \in \Psi_{\operatorname{sus}(2)}^{-\infty}(Z ; E)\left[\epsilon / \epsilon^{2}\right]
$$

with $a_{0}, a_{1} \in \Psi_{\operatorname{sus}(2)}^{-\infty}(Z ; E)$. A special case of Lemma 5 below shows that this is a trace functional

$$
\operatorname{Tr}_{\mathrm{ad}}(a * b-b * a)=0, \quad \forall a, b \in \Psi_{\operatorname{sus}(2)}^{-\infty}(Z ; E)\left[\epsilon / \epsilon^{2}\right] .
$$


Consider the 1-form

$$
\alpha(a)=\operatorname{Tr}_{\mathrm{ad}}\left(a^{-1} * d a\right) \text { on } G^{-\infty}(Z ; E)\left[\epsilon / \epsilon^{2}\right],
$$

where the inverse of $a \in G_{\operatorname{sus}(2)}^{-\infty}(Z ; E)\left[\epsilon / \epsilon^{2}\right]$ is with respect to the the truncated $*$-product

$$
a^{-1}=a_{0}^{-1}-\epsilon a_{0}^{-1}\left(a_{1}+\frac{1}{2 i}\left(\frac{\partial a_{0}}{\partial t} a_{0}^{-1} \frac{\partial a_{0}}{\partial \tau}-\frac{\partial a_{0}}{\partial \tau} a_{0}^{-1} \frac{\partial a_{0}}{\partial t}\right)\right) a_{0}^{-1} .
$$

The adiabatic determinant is then defined by

$$
\operatorname{det}_{\mathrm{ad}}(g)=\exp \left(\int_{[0,1]} \gamma^{*} \alpha\right)
$$

where $\gamma ;[0,1] \longrightarrow G_{\operatorname{sus}(2)}^{-\infty}(Z ; E)\left[\epsilon / \epsilon^{2}\right]$ is any smooth path from the identity to $g$. Since the integral of $\alpha$ along a loop gives an integer multiple of $2 \pi i$ (see for instance proposition 4.4 in [24]), this definition does not depend on the choice of $\gamma$. From (10.5),

$$
\alpha(a b)=\operatorname{Tr}_{\mathrm{ad}}\left((a * b)^{-1} d(a * b)\right)=\operatorname{Tr}_{\mathrm{ad}}\left(a^{-1} * d a\right)+\operatorname{Tr}_{\mathrm{ad}}\left(b^{-1} * d b\right),
$$

and hence

$$
m^{*} \alpha=\pi_{L}^{*} \alpha+\pi_{R}^{*} \alpha
$$

where

$$
\begin{aligned}
m: G_{\operatorname{sus}(2)}^{-\infty}(Z ; E)\left[\epsilon / \epsilon^{2}\right] \times G_{\operatorname{sus}(2)}^{-\infty}(Z ; E)\left[\epsilon / \epsilon^{2}\right] \ni(a, b) & \\
& \longmapsto a[*] b \in G_{\operatorname{sus}(2)}^{-\infty}(Z ; E)\left[\epsilon / \epsilon^{2}\right] .
\end{aligned}
$$

is the composition given by the truncated $*$-product while $\pi_{L}$ and $\pi_{R}$ are the projections on the left and right factor. The multiplicativity of the adiabatic determinant follows directly from (10.10).

\section{The determinant line bundle}

We next describe the construction, and especially primitivity, of the determinant line bundle over a smooth classifying group for even K-theory.

Definition 3. A primitive line bundle over a (Fréchet-Lie) group

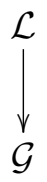


is a smooth, and locally trivial, line bundle equipped with an isomorphism of the line bundles

$$
\begin{gathered}
\pi_{L}^{*} \mathcal{L} \otimes \pi_{R}^{*} \mathcal{L} \stackrel{\simeq}{\longrightarrow} m^{*} \mathcal{L} \text { over } \mathcal{G} \times \mathcal{G}, \\
\pi_{L}: \mathcal{G} \times \mathcal{G} \ni(a, b) \longrightarrow a \in \mathcal{G}, \quad \pi_{R}: \mathcal{G} \times \mathcal{G} \ni(a, b) \longrightarrow b \in \mathcal{G}, \\
m: \mathcal{G} \times \mathcal{G} \ni(a, b) \longrightarrow a b \in \mathcal{G}
\end{gathered}
$$

which is associative in the sense that for any three elements, $a, b, c \in \mathcal{G}$, the two induced isomorphisms

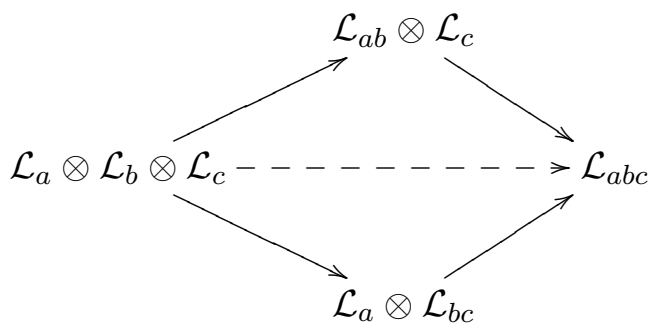

are the same.

For the reduced classifying group, $G_{\text {sus,ind }=0}^{-\infty}(Z ; E)$, a construction of the determinant line bundle, with this primitivity property, was given in [25], although only in the 'geometric case'. A variant of the construction there, also depending heavily on the properties of the suspended determinant but using instead the 'dressed' delooping sequence (for the loop group)

$$
G_{\operatorname{sus}(2)}^{-\infty}(Z ; E)\left[\epsilon / \epsilon^{2}\right] \longrightarrow \tilde{G}_{\operatorname{sus}(2)}^{-\infty}(Z ; E)\left[\epsilon / \epsilon^{2}\right] \longrightarrow G_{\text {sus,ind }=0}^{-\infty}(Z ; E)
$$

again constructs the determinant line bundle, with primitivity condition, over the component of the identity in the loop group. In this section, by modifying an idea from the book of Pressley and Segal, [29], we show how to extend this primitive line bundle to the whole of the classifying group.

In (11.4) the central, contractible, group is based on the half-open but smooth-flat loop group:

$$
\begin{aligned}
& \tilde{G}_{\operatorname{sus}(2)}^{-\infty}(Z ; E)=\left\{\tilde{a}: \mathbb{R}_{(t, \tau)}^{2} \longrightarrow \Psi^{-\infty}(Z ; E) ; \lim _{t \rightarrow-\infty} \tilde{a}(t, \tau)=0,\right. \\
& \left.\quad \frac{\partial \tilde{a}}{\partial t} \in \mathcal{S}\left(\mathbb{R}^{2} ; \Psi^{-\infty}(Z ; E)\right), \tilde{a}(t, \tau), \tilde{a}(\infty, \tau) \in G^{-\infty}(Z ; E) \forall t, \tau \in \mathbb{R}\right\} .
\end{aligned}
$$

Note that automatically, $\lim _{\tau \rightarrow \infty} \tilde{a}(t, \tau)=0$ for all $t \in[-\infty, \infty]$. This group has an extension with the product having the same 'correction term' given by the Poisson bracket on $\mathbb{R}^{2}$ as in (10.1):

$$
\tilde{G}_{\operatorname{sus}(2)}^{-\infty}(Z ; E)\left[\epsilon / \epsilon^{2}\right]=\tilde{G}_{\operatorname{sus}(2)}^{-\infty}(Z ; E) \oplus \Psi_{\operatorname{sus}(2)}^{-\infty}(Z ; E)
$$


where the additional terms at level $\epsilon$ are just Schwartz functions valued in the smoothing operators without any additional invertibility. Note that the term in the product involving the Poisson bracket always leads to a Schwartz function on $\mathbb{R}^{2}$, since one factor is differentiated with respect to $t$. Thus $\tilde{G}_{\operatorname{sus}(2)}^{-\infty}(Z ; E)\left[\epsilon / \epsilon^{2}\right]$ is again contractible, with just the addition of a lower order 'affine' term.

To expand the quotient group to the whole classifying group, choose one element $s \in G_{\text {sus }}^{-\infty}(Z ; E)$ of index 1 . Then $s^{j}$ is in the component of index $j$ so each element $a \in G_{\text {sus }}^{-\infty}(Z ; E)$ can be connected by a curve, and hence a flat-smooth loop, to $s^{j}$ for precisely one $j$. The group in (11.5) may then be further enlarged to

$$
\begin{aligned}
& \mathcal{D}_{\operatorname{sus}(2)}^{-\infty}(Z ; E)=\left\{\tilde{a}: \mathbb{R}_{(t, \tau)}^{2} \longrightarrow \Psi^{-\infty}(Z ; E) ; \frac{\partial \tilde{a}}{\partial t} \in \mathcal{S}\left(\mathbb{R}^{2} ; \Psi^{-\infty}(Z ; E)\right),\right. \\
& \left.\quad \lim _{t \rightarrow-\infty} \tilde{a}(t, \tau)=s^{j} \text { for some } j, \tilde{a}(t, \tau), \tilde{a}(\infty, \tau) \in G^{-\infty}(Z ; E) \forall t, \tau \in \mathbb{R}\right\} .
\end{aligned}
$$

This expanded group has countably many components, labelled by $j$, and the restriction map to $t=\infty$ is a surjection to $G_{\text {sus }}^{-\infty}(Z ; E)$. Thus, after adding the same affine lower order terms, (11.4) is replaced by the new short exact sequence

$$
G_{\operatorname{sus}(2)}^{-\infty}(Z ; E)\left[\epsilon / \epsilon^{2}\right] \longleftrightarrow \mathcal{D}_{\operatorname{sus}(2)}^{-\infty}(Z ; E)\left[\epsilon / \epsilon^{2}\right] \stackrel{\tilde{R}_{\infty}}{\longrightarrow} G_{\text {sus }}^{-\infty}(Z ; E) .
$$

The central group is no longer contractible, although each of its connected component is. However the 1 -form $\alpha$ in (10.6) can be extended to give a smooth 1 -form on $\mathcal{D}_{\operatorname{sus}(2)}^{-\infty}(Z ; E)\left[\epsilon / \epsilon^{2}\right]$. Indeed, the adiabatic trace has an obvious extension to a functional on

$$
\widetilde{\Psi}_{\operatorname{sus}(2)}^{-\infty}(Z ; E)\left[\epsilon / \epsilon^{2}\right]=\widetilde{\Psi}_{\operatorname{sus}(2)}^{-\infty}(Z ; E) \oplus \Psi_{\operatorname{sus}(2)}^{-\infty}(Z ; E)
$$

where

$$
\begin{aligned}
& \widetilde{\Psi}_{\operatorname{sus}(2)}^{-\infty}(Z ; E)=\left\{a \in \mathcal { C } ^ { \infty } \left(\mathbb{R}^{2} ; \Psi^{-\infty}(Z ; E) ; \frac{\partial a}{\partial t} \in \mathcal{S}\left(\mathbb{R}^{2} ; \Psi^{-\infty}(Z ; E)\right),\right.\right. \\
& \left.\lim _{t \rightarrow-\infty} a(t, \tau)=0\right\} .
\end{aligned}
$$

Namely

$$
\widetilde{\operatorname{Tr}}_{\mathrm{ad}}(a)=\frac{1}{2 \pi} \int_{\mathbb{R}^{2}} \operatorname{Tr}_{Z}\left(a_{1}\right) d t d \tau, a=a_{0}+\epsilon a_{1} \in \widetilde{\Psi}_{\operatorname{sus}(2)}^{-\infty}(Z ; E)\left[\epsilon / \epsilon^{2}\right]
$$

Thus, on $\mathcal{D}_{\operatorname{sus}(2)}^{-\infty}(Z ; E)\left[\epsilon / \epsilon^{2}\right]$, one can consider the smooth 1-form

$$
\widetilde{\alpha}(a)=\frac{1}{2} \widetilde{\operatorname{Tr}}_{\mathrm{ad}}\left(a^{-1} * d a+d a * a^{-1}\right)
$$

which restricts to $\alpha$ on $G_{\operatorname{sus}(2)}^{-\infty}(Z ; E)\left[\epsilon / \epsilon^{2}\right]$. 
Lemma 5. For $a=a_{0}+\epsilon a_{1}$ and $b=b_{0}+\epsilon b_{1}$ in $\widetilde{\Psi}_{\operatorname{sus}(2)}^{-\infty}(Z ; E)\left[\epsilon / \epsilon^{2}\right]$,

$$
\widetilde{\operatorname{Tr}}_{\mathrm{ad}}(a * b-b * a)=\frac{1}{2 \pi i} \int_{\mathbb{R}} \operatorname{Tr}_{Z}\left(\frac{\partial a_{0}}{\partial \tau}(\infty, \tau) b_{0}(\infty, \tau)\right) d \tau .
$$

In particular, this trace-defect vanishes if $a, b \in \Psi_{\operatorname{sus}(2)}^{-\infty}(Z ; E)\left[\epsilon / \epsilon^{2}\right]$.

Proof. By definition of the truncated $*$-product and using the trace property of $\operatorname{Tr}_{Z}$,

$$
\widetilde{\operatorname{Tr}}_{\mathrm{ad}}(a * b-b * a)=-\frac{1}{2 \pi i} \int_{\mathbb{R}^{2}} \operatorname{Tr}_{Z}\left(\frac{\partial a_{0}}{\partial t} \frac{\partial b_{0}}{\partial \tau}-\frac{\partial a_{0}}{\partial \tau} \frac{\partial b_{0}}{\partial t}\right) d t d \tau .
$$

Integrating by parts the first term on the right,

$$
\begin{aligned}
\int_{\mathbb{R}^{2}} \operatorname{Tr}_{Z}\left(\frac{\partial a_{0}}{\partial t} \frac{\partial b_{0}}{\partial \tau}\right) d t d \tau= & -\int_{\mathbb{R}^{2}} \operatorname{Tr}_{Z}\left(\frac{\partial^{2} a_{0}}{\partial \tau \partial t} b_{0}\right) d t d \tau \\
= & -\int_{\mathbb{R}^{2}} \frac{\partial}{\partial t}\left(\operatorname{Tr}_{Z}\left(\frac{\partial a_{0}}{\partial \tau} b_{0}\right)\right) d t d \tau \\
& +\int_{\mathbb{R}^{2}} \operatorname{Tr}_{Z}\left(\frac{\partial a_{0}}{\partial \tau} \frac{\partial b_{0}}{\partial t}\right) d t d \tau
\end{aligned}
$$

Thus,

$$
\begin{aligned}
\operatorname{Tr}_{\text {ad }}(a * b-b * a) & =\frac{1}{2 \pi i} \int_{\mathbb{R}^{2}} \frac{\partial}{\partial t}\left(\operatorname{Tr}_{Z}\left(\frac{\partial a_{0}}{\partial \tau} b_{0}\right)\right) d t d \tau \\
& =\frac{1}{2 \pi i} \int_{\mathbb{R}} \operatorname{Tr}_{Z}\left(\frac{\partial a_{0}}{\partial \tau}(\infty, \tau) b_{0}(\infty, \tau)\right) d \tau
\end{aligned}
$$

Proposition 9. Under the maps on the product

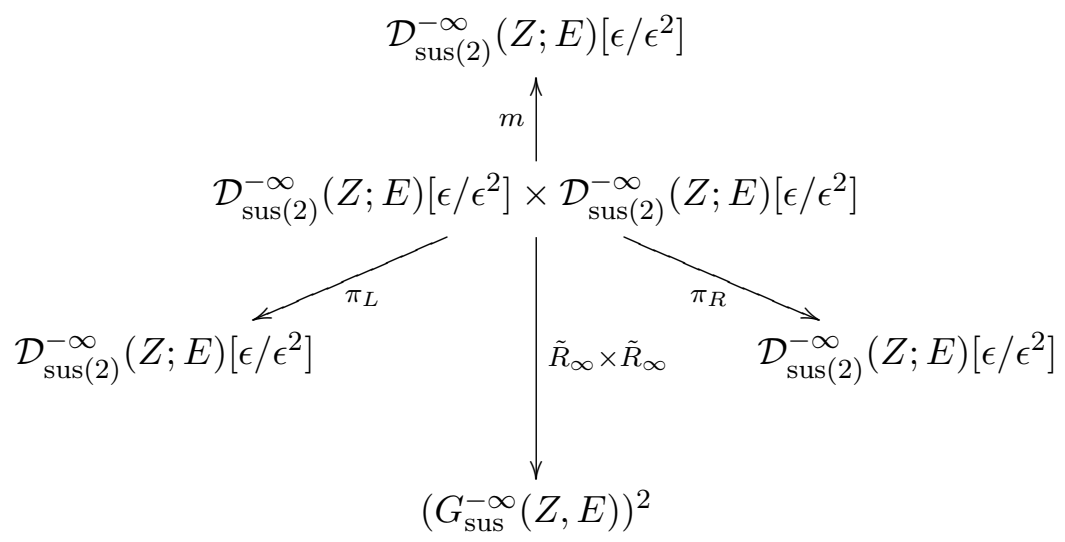

the 1-form $\tilde{\alpha}$ in (11.12) satisfies

$$
m^{*} \tilde{\alpha}=\pi_{L}^{*} \tilde{\alpha}+\pi_{R}^{*} \tilde{\alpha}+\left(\tilde{R}_{\infty} \times \tilde{R}_{\infty}\right)^{*} \delta
$$


with

$$
\delta(a, b)=-\frac{1}{4 \pi i} \int_{\mathbb{R}} \operatorname{Tr}_{Z}\left(a^{-1}(d a) \frac{\partial b}{\partial \tau} b^{-1}-(d b) b^{-1} a^{-1} \frac{\partial a}{\partial \tau}\right) d \tau
$$

on $G_{\mathrm{sus}}^{-\infty}(Z ; E) \times G_{\mathrm{sus}}^{-\infty}(Z ; E)$.

Proof. If $a, b \in \mathcal{D}_{\operatorname{sus}(2)}^{-\infty}(Z ; E)\left[\epsilon / \epsilon^{2}\right]$, the trace-defect formula of Lemma 5 gives

$$
\begin{aligned}
\widetilde{\operatorname{Tr}}_{\mathrm{ad}} & \left((a * b)^{-1} * d(a * b)\right) \\
= & \widetilde{\operatorname{Tr}}_{\mathrm{ad}}\left(b^{-1} * a^{-1} * d a * b+b^{-1} * d b\right) \\
= & \widetilde{\operatorname{Tr}}_{\mathrm{ad}}\left(a^{-1} * d a\right)+\widetilde{\operatorname{Tr}}_{\mathrm{ad}}\left(b^{-1} * d b\right) \\
& +\widetilde{\operatorname{Tr}}_{\mathrm{ad}}\left(b^{-1} *\left(a^{-1} * d a * b\right)-\left(a^{-1} * d a * b\right) * b^{-1}\right) \\
= & \widetilde{\operatorname{Tr}}_{\mathrm{ad}}\left(a^{-1} * d a\right)+\widetilde{\operatorname{Tr}}_{\mathrm{ad}}\left(b^{-1} * d b\right) \\
& +\frac{1}{2 \pi i} \int_{\mathbb{R}} \operatorname{Tr}_{Z}\left(\frac{\partial b_{0}^{-1}}{\partial \tau}(\infty, \tau)\left(a_{0}^{-1} d a_{0} b_{0}\right)(\infty, \tau)\right) d \tau \\
= & \widetilde{\operatorname{Tr}}_{\mathrm{ad}}\left(a^{-1} * d a\right)+\widetilde{\operatorname{Tr}}_{\mathrm{ad}}\left(b^{-1} * d b\right) \\
& -\frac{1}{2 \pi i} \int_{\mathbb{R}} \operatorname{Tr}_{Z}\left(\frac{\partial b_{0}}{\partial \tau}(\infty, \tau) b_{0}^{-1}(\infty, \tau) a_{0}^{-1}(\infty, \tau) d a_{0}(\infty, \tau)\right) d \tau
\end{aligned}
$$

Similarly,

$$
\begin{aligned}
& \widetilde{\operatorname{Tr}}_{\mathrm{ad}}\left(d(a * b) *(a * b)^{-1}\right) \\
& =\widetilde{\operatorname{Tr}}_{\mathrm{ad}}\left(d a * a^{-1}\right)+\widetilde{\operatorname{Tr}}_{\mathrm{ad}}\left(d b * b^{-1}\right) \\
& \quad+\frac{1}{2 \pi i} \int_{\mathbb{R}} \operatorname{Tr}_{Z}\left(d b_{0}(\infty, \tau) b^{-1}(\infty, \tau) a^{-1}(\infty, \tau) \frac{\partial a_{0}}{\partial \tau}(\infty, \tau)\right) d \tau
\end{aligned}
$$

Combining these two computations, the result follows.

Proposition 10. The adiabatic determinant on the normal subgroup in (11.8) induces the determinant line bundle, $\mathcal{L}$, which is primitive over the quotient and $\tilde{\alpha}$ in (11.12) defines a connection $\nabla_{\mathrm{ad}}$ on $\mathcal{L}$ with curvature form the 2-form part of the universal even Chern character of (2.22).

Proof. The form $\tilde{\alpha}$ in (11.12) restricts to $\alpha$ in $(10.6)$ on $G_{\operatorname{sus}(2)}^{-\infty}(Z ; E)$ $\left[\epsilon / \epsilon^{2}\right]$. The latter is the differential of the logarithm of $\operatorname{det}_{\mathrm{ad}}$. As a special case of Proposition 9 above, the first factor may be restricted to $G_{\operatorname{sus}(2)}^{-\infty}(Z ; E)$, and then $\delta$ in (11.17) vanishes since $a \equiv 0$. This shows that as a connection on the trivial bundle over $\mathcal{D}_{\operatorname{sus}(2)}^{-\infty}(Z ; E)\left[\epsilon / \epsilon^{2}\right], d-\tilde{\alpha}$ is invariant under the left action of $G_{\operatorname{sus}(2)}^{-\infty}(Z ; E)\left[\epsilon / \epsilon^{2}\right]$, acting through the adiabatic determinant on the fibres. Thus $d-\tilde{\alpha}$ projects to a connection $\nabla_{\mathrm{ad}}$ on the determinant line bundle over $G_{\text {sus }}^{-\infty}(Z ; E)$ defined as the quotient by this action, i.e. as the line bundle induced by $\operatorname{det}_{\mathrm{ad}}$ as a representation of the structure group. 
To compute the curvature we simply need to compute the differential of $\tilde{\alpha}$. Using the trace-defect formula of Lemma 5,

$$
\begin{aligned}
& d \widetilde{\operatorname{Tr}}_{\mathrm{ad}}\left(a^{-1} * d a\right) \\
& =-\widetilde{\operatorname{Tr}}_{\mathrm{ad}}\left(a^{-1} * d a * a^{-1} * d a\right)=-\frac{1}{2} \widetilde{\operatorname{Tr}}_{\mathrm{ad}}\left(\left[a^{-1} * d a, a^{-1} * d a\right]\right), \\
& =-\frac{1}{4 \pi i} \int_{\mathbb{R}} \operatorname{Tr}_{Z}\left(\left(\frac{\partial}{\partial \tau}\left(\sigma^{-1} d \sigma\right)\right) \sigma^{-1} d \sigma\right) d \tau, \text { with } \sigma=a_{0}(\infty, \tau), \\
& \quad=\frac{1}{4 \pi i} \int_{\mathbb{R}} \operatorname{Tr}_{Z}\left(\sigma^{-1} \frac{\partial \sigma}{\partial \tau}\left(\sigma^{-1} d \sigma\right)^{2}-\sigma^{-1} d\left(\frac{\partial \sigma}{\partial \tau}\right) \sigma^{-1} d \sigma\right) d \tau
\end{aligned}
$$

Similarly, we compute that

$$
\begin{aligned}
& d \widetilde{\operatorname{Tr}}_{\mathrm{ad}}\left(d a * a^{-1}\right) \\
& =\widetilde{\operatorname{Tr}}_{\mathrm{ad}}\left(d a * a^{-1} * d a * a^{-1}\right)=\frac{1}{2} \widetilde{\operatorname{Tr}}_{\mathrm{ad}}\left(\left[d a * a^{-1}, d a * a^{-1}\right]\right), \\
& \quad=\frac{1}{4 \pi i} \int_{\mathbb{R}} \operatorname{Tr}_{Z}\left(\left(\frac{\partial}{\partial \tau}\left(d \sigma \sigma^{-1}\right)\right) d \sigma \sigma^{-1}\right) d \tau \\
& \quad=-\frac{1}{4 \pi i} \int_{\mathbb{R}} \operatorname{Tr}_{Z}\left(d \sigma \sigma^{-1} \frac{\partial \sigma}{\partial \tau} \sigma^{-1} d \sigma \sigma^{-1}-d\left(\frac{\partial \sigma}{\partial \tau}\right) \sigma^{-1} d \sigma \sigma^{-1}\right) d \tau
\end{aligned}
$$

Recall that the 2 -form part of the universal even Chern character on $G_{\text {sus }}^{-\infty}$ $(Z ; E)$ is given by (cf. formula $(3.7)$ in $[\mathbf{2 3}])$

$$
\left(\mathrm{Ch}_{\text {even }}\right)_{[2]}=\frac{1}{2(2 \pi i)^{2}} \int_{\mathbb{R}} \operatorname{Tr}_{Z}\left(\sigma^{-1} \frac{\partial \sigma}{\partial \tau}\left(\sigma^{-1} d \sigma\right)^{2}\right) d \tau \text {. }
$$

Thus, combining (11.20) and (11.21),

$$
d \tilde{\alpha}=\frac{1}{4 \pi i} \int_{\mathbb{R}} \operatorname{Tr}_{Z}\left(\sigma^{-1} \frac{\partial \sigma}{\partial \tau}\left(\sigma^{-1} d \sigma\right)^{2}\right) d \tau=2 \pi i \tilde{R}_{\infty}^{*}\left(\left(\mathrm{Ch}_{\text {even }}\right)_{[2]}\right),
$$

that is,

$$
\frac{i}{2 \pi} \nabla_{\mathrm{ad}}^{2}=\tilde{R}_{\infty}^{*}\left(\left(\mathrm{Ch}_{\mathrm{even}}\right)_{[2]}\right)
$$

Next, this construction of the determinant bundle is extended to the geometric case. The sequence, (11.8), being natural, extends to give smooth bundles over the fibres of (1):

$$
G_{\operatorname{sus}(2)}^{-\infty}(\phi ; E)\left[\epsilon / \epsilon^{2}\right] \hookrightarrow \mathcal{D}_{\operatorname{sus}(2)}^{-\infty}(\phi ; E)\left[\epsilon / \epsilon^{2}\right] \stackrel{\tilde{R}_{\infty}}{\longrightarrow} G_{\text {sus }}^{-\infty}(\phi ; E) .
$$

Furthermore, using the connection chosen earlier, the form $\tilde{\alpha}$ in (11.12) can be replaced by $\tilde{\alpha}_{\phi}$ by substituting $\nabla^{\phi, E}$ for $d$ throughout. The resulting 1-form is well-defined on $\mathcal{D}_{\operatorname{sus}(2)}^{-\infty}(\phi ; E)$. Moreover the proof of proposition 9 only depends on the derivation property of $d$ so extend directly to $\tilde{\alpha}_{\phi}$. In 
particular (11.17) carries over to the fibre products. This leads to the following geometric version of Proposition 10.

Proposition 11. The adiabatic determinant on the fibres of the structure bundle in (11.8) induces the determinant line bundle, $\mathcal{L}$, over $G_{\text {sus }}^{-\infty}$ $(\phi ; E) ;$ the 1-form $\tilde{\alpha}_{\phi}$ defines a connection $\nabla_{\phi}$ on $\mathcal{L}$ with curvature the 2 -form part of the even Chern character on $G_{\text {sus }}^{-\infty}(\phi ; E)$.

Proof. What is slightly different in the geometric case is the computation of the curvature of $\nabla_{\phi}$. Taking into account (4.6), the analogue of (11.20) and (11.21) is

$$
\begin{aligned}
d \tilde{\alpha}_{\phi}= & \frac{1}{4 \pi i} \int_{\mathbb{R}} \operatorname{Tr}_{Z}\left(\sigma^{-1} \frac{\partial \sigma}{\partial \tau}\left(\sigma^{-1} \nabla^{\phi, E} \sigma\right)^{2}\right) d \tau \\
& +\frac{1}{2} \operatorname{Tr}_{\mathrm{ad}}\left(a^{-1} *([\omega, a])+([\omega, a]) * a^{-1}\right)
\end{aligned}
$$

where $\sigma=a_{0}(\infty, \tau)$. To compute the second term, we use the identity $a^{-1} *$ $(a \omega)=(\omega a) * a^{-1}=\omega$ to rewrite it as

$$
\begin{aligned}
\frac{1}{2} \operatorname{Tr}_{\mathrm{ad}}\left(a^{-1} *([\omega, a])+([\omega, a]) * a^{-1}\right)= & \frac{1}{2} \operatorname{Tr}_{\mathrm{ad}}\left(a^{-1} *(\omega a)-(\omega a) * a^{-1}\right) \\
& +\frac{1}{2} \operatorname{Tr}_{\mathrm{ad}}\left(a^{-1} *(a \omega)-(a \omega) * a^{-1}\right) .
\end{aligned}
$$

Using the trace-defect formula of lemma 5 , this gives

$$
\begin{aligned}
d \tilde{\alpha}_{\phi} & =\frac{1}{4 \pi i} \int_{\mathbb{R}} \operatorname{Tr}_{Z}\left(\sigma^{-1} \frac{\partial \sigma}{\partial \tau}\left(\left(\sigma^{-1} \nabla^{\phi, E} \sigma\right)^{2}-\sigma^{-1} \omega \sigma-\omega\right)\right) d \tau \\
& =2 \pi i \tilde{R}_{\infty}^{*}\left(\left(\mathrm{Ch}_{\mathrm{even}}\left(\nabla^{\phi, E}\right)\right)_{[2]}\right),
\end{aligned}
$$

from which the result follows.

\section{The K-theory gerbe}

First we consider the universal K-theory gerbe, i.e. the gerbe over the classifying space $G^{-\infty}(Z ; E)$ for odd K-theory. Such a gerbe was originally introduced by Carey and Mickelsson $[\mathbf{9}],[\mathbf{1 0}]$ over a slightly different classifying space for odd K-theory, namely the space of unitary operators which are perturbations of the identity by operators of trace class. We propose a different construction of the universal K-theory gerbe using the determinant line bundle of proposition 10 . 
Recall that the delooping sequence (5) for a single manifold

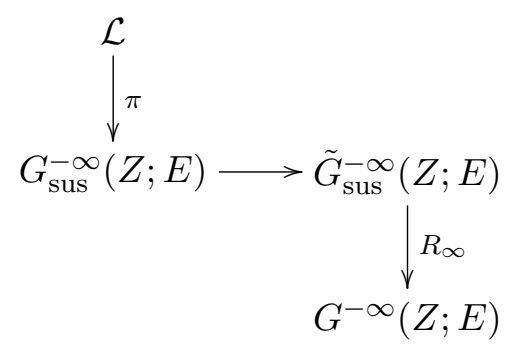

is a classifying sequence for K-theory, the normal subgroup is classifying for even K-theory, the central group is contractible and the quotient is classifying for odd K-theory. Moreover, in the preceding section, we have constructed the smooth primitive determinant line bundle over $G_{\text {sus }}^{-\infty}(Z ; E)$ with connection $\nabla_{\text {ad }}$ given in Proposition 10. This induces the K-theory gerbe over the classifying space, as a line bundle over the fibre product of two copies of the fibration $R_{\infty}$ :

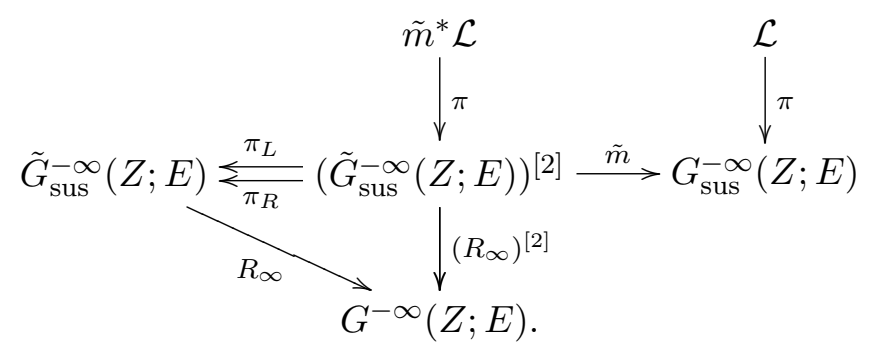

Here $\tilde{m}:\left(\tilde{G}_{\text {sus }}^{-\infty}(Z ; E)\right)^{[2]} \longrightarrow G_{\text {sus }}^{-\infty}(Z ; E)$ is the fibre-shift map $\tilde{m}(a, b)=$ $a b^{-1}$, where $R_{\infty}(a)=R_{\infty}(b)$, by definition of the fibre product, so $\tilde{m}(a, b) \in$ $G_{\text {sus }}^{-\infty}(Z ; E)$ by the exactness of $(12.1)$, as indicated.

THEOREM 2. There is a connection $\tilde{\nabla}_{\mathrm{ad}}$ on $\tilde{m}^{*} \mathcal{L}$ with curvature

$$
\frac{i}{2 \pi} F_{\tilde{\nabla}_{\mathrm{ad}}}=\pi_{L}^{*} \tilde{\eta}_{2}-\pi_{R}^{*} \tilde{\eta}_{2} \text { on }\left(\tilde{G}_{\mathrm{sus}}^{-\infty}(Z ; E)\right)^{[2]}
$$

where the B-field, $\tilde{\eta}_{2}$, is the 2-form part of the eta form in (3.2) which has basic differential the 3-form part of the odd Chern character on $G^{-\infty}(Z ; E)$, as shown by (3.6).

Proof. The connection $\nabla_{\text {ad }}$ on $\mathcal{L}$ as a bundle over $G_{\text {sus }}^{-\infty}(Z ; E)$ given by Proposition 10 pulls back to a connection $\tilde{m}^{*} \nabla_{\text {ad }}$ on $\tilde{m}^{*} \mathcal{L}$. The curvature is just the pull-back of the curvature on $G_{\text {sus }}^{-\infty}(Z ; E)$ and again by Proposition 10 this is the 2-form part of the Chern character. By Proposition 2, the 2 -form part of the eta form on $\tilde{G}_{\text {sus }}^{-\infty}(Z ; E)$ pulls back under the product map as in (3.5). To apply this result here we need to invert the right factor, 
to change from $m$ to $\tilde{m}$, which also has the effect of changing the sign of the eta form from that factor leading to

$$
\tilde{m}^{*} \tilde{\eta}=\pi_{L}^{*} \tilde{\eta}-\pi_{R}^{*} \tilde{\eta}+d\left(\tilde{\delta}_{\text {odd }}^{\prime}\right)+\left(R_{\infty} \times R_{\infty}\right)^{*} \delta_{\text {even }}^{\prime}
$$

where the primes indicate that the forms are first pulled back under inversion in the second variable. Now, restricting (12.4) to the fibre diagonal gives

$$
\tilde{m}^{*} \mathrm{Ch}_{\mathrm{even}}=\pi_{L}^{*} \tilde{\eta}-\pi_{R}^{*} \tilde{\eta}+d\left(\tilde{\delta}_{\text {odd }}^{\prime}\right)
$$

since the last term now factors through the constant map to the identity. This corresponds to the middle row of (12.2). In particular, if the connection is modified by the 1 -form part of $\delta_{\text {odd }}^{\prime}$

$$
\tilde{\nabla}_{\text {ad }}=\tilde{m}^{*} \nabla_{\text {ad }}+2 \pi i\left(\tilde{\delta}_{\text {odd }}^{\prime}\right)_{1}
$$

then it has curvature as claimed

$$
\frac{i}{2 \pi}\left(\tilde{\nabla}_{\mathrm{ad}}\right)^{2}=\tilde{m}^{*}\left(\mathrm{Ch}_{\mathrm{even}}\right)_{2}-d\left(\delta_{\text {odd }}^{\prime}\right)_{1}=\pi_{L}^{*} \tilde{\eta}_{2}-\pi_{R}^{*} \tilde{\eta}_{2}
$$

which is precisely the statement that $\tilde{\eta}_{2}$ is a B-field for the gerbe. The curving of the gerbe is then the basic form of which the differential of the B-field is the pull-back and from (3.6)

$$
d \tilde{\eta}_{2}=R_{\infty}^{*}\left(\mathrm{Ch}_{\mathrm{odd}}\right)_{3} .
$$

The bundle gerbe (12.2) with connection given by theorem 2 is universal in the sense that given an odd K-theory class $[g] \in K^{1}(Y)$ represented by a smooth map $g: Y \rightarrow G^{-\infty}(Z ; E)$, the pull-back of (12.2) to $Y$ by $g$ gives a bundle gerbe (with connection) on $Y$ whose curving 3 -form is given by $g^{*} \mathrm{Ch}_{\text {odd }}$ (cf. Theorem 5.1 in $[\mathbf{1 3}]$ ).

Since

$$
\left(\mathrm{Ch}_{\mathrm{odd}}\right)_{3}=\frac{1}{6(2 \pi i)^{2}} \operatorname{Tr}\left(\left(\sigma^{-1} d \sigma\right)^{3}\right)
$$

is the image in $H^{3}\left(G^{-\infty}(Z ; E) ; \mathbb{C}\right)$ of the generator of $H^{3}\left(G^{-\infty}(Z ; E) ; \mathbb{Z}\right) \cong$ $\mathbb{Z}$, we also note that the bundle gerbe $(12.2)$ is basic for the group $G^{-\infty}$ $(Z ; E)$. By considering an $n$-dimensional subspace of $L^{2}(Z ; E)$ with norm defined by a choice of metric on $Z$ and of Hermitian metric on $E$, we get a natural inclusion $\mathrm{U}(n) \subset G^{-\infty}(Z ; E)$. Pulling back $(12.2)$ to $\mathrm{U}(n)$ via this inclusion gives the basic bundle gerbe $\mathrm{U}(n)$. This is a 'smooth' construction in any reasonable sense although it is infinite dimensional in nature.

Infinite dimensional constructions of the basic bundle gerbe of a Lie group first appeared in the book of Brylinski [6] and later in [7]. The tautological bundle gerbe of Murray [26] for 2-connected manifolds also provides such a construction for simply connected Lie groups (see also [12]). More recently, finite dimensional constructions of the basic gerbe were obtained by Gawedzki and Reis [14] for $\mathrm{SU}(n)$ and shortly after by Meinrenken [19] 
for simple simply connected Lie groups. The construction of Meinrenken was subsequently generalized to non-simply connected Lie groups in [15] and $[\mathbf{2 7}]$.

\section{Geometric gerbe for an odd elliptic family}

As pointed out in $[\mathbf{1 1}]$, gerbes are intimately related to index theory. In our case, we have the following construction of the index gerbe (with connection) associated to a family of self-adjoint elliptic pseudodifferential operators and more generally to a product-type family of fully elliptic operators.

TheOREM 3. Let $A \in \Psi^{1}(M / Y ; E)$ be a self-adjoint elliptic family as in Section 6 , or a product-type fully elliptic family $A(t) \in \Psi_{\mathrm{ps}}^{m, l}(M / Y ; E)$ then the determinant bundle induces a bundle-gerbe

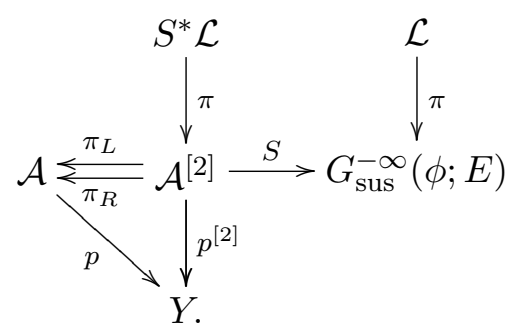

The connection on $S^{*} \mathcal{L}$

$$
\nabla_{\mathcal{A}}=S^{*} \nabla+\gamma, \gamma=2 \pi i\left(\tilde{\delta}_{\mathcal{A}}\right)_{1}
$$

given by the 1-form part of the form in (6.17), is primitive in the sense that the curvature on $\mathcal{A}^{[2]}$ splits

$$
\frac{i}{2 \pi}\left(\nabla_{\mathcal{A}}\right)^{2}=\pi_{L}^{*} \eta_{\mathcal{A}, 2}-\pi_{R}^{*} \eta_{\mathcal{A}, 2}
$$

showing that $\eta_{\mathcal{A}, 2}$ is a B-field and that the gerbe has curving 3-form

$$
d \eta_{\mathcal{A}, 2}=p^{*} \mathrm{Ch}_{\mathcal{A}, 3}
$$

the 3-form part of the Chern character of the index bundle of the family.

Proof. This result follows by an argument parallel to the preceding one, given (6.17), Proposition 10, Proposition 11 and the 3-form part of $(8.4)$.

This result can be seen as a pseudodifferential generalization of a result of Lott (Theorem 1 in $[\mathbf{1 8}]$ ). See also [8] for a different treatment of the index gerbe and $[\mathbf{1 3}]$ for a generalization of $([\mathbf{1 8}]$, Theorem 1$)$ to families of Dirac operators on odd dimensional manifolds with boudary. 


\section{Relation with the Bismut-Cheeger eta form}

Amongst the most important geometric examples of self-adjoint elliptic operators are the Dirac-type operators on odd dimensional manifolds. Suppose now that the fibres of the fibration (1) are odd dimensional. Let $g \in$ $\mathcal{C}^{\infty}\left(M ; \odot^{2} T^{*}(M / Y)\right)$ be a family of fibrewise metrics and let $\mathbb{C} \ell(T(M / Y))$ be the associated bundle of Clifford algebras for the vertical tangent bundle $T(M / Y)$. Let $\mathbb{E} \rightarrow M$ be a Clifford module with respect to $\mathbb{C} \ell(T(M / Y))$ with Clifford action $c: \mathbb{C} \ell(T(M / Y)) \rightarrow \operatorname{End}(\mathbb{E})$. Let also $\nabla^{\mathbb{E}}$ be a family of fibrewise Clifford connections, that is a family of unitary connections such that

$\left[\nabla_{X_{1}}^{\mathbb{E}}, c\left(X_{2}\right)\right]=c\left(\nabla_{X_{1}}^{L C} X_{2}\right) \in \mathcal{C}^{\infty}(M ; \operatorname{End}(\mathbb{E})), \forall X_{1}, X_{2} \in \mathcal{C}^{\infty}(M ; T(M / Y))$, where $\nabla^{L C}$ is the fibrewise Levi-Civita connection associated to the family of metrics $g$. This data allows us to define a family of Dirac-type operators by

$$
\check{\partial}=c \circ \nabla^{\mathbb{E}} \text {. }
$$

For invertible families of this type, Bismut and Cheeger introduced in $[\mathbf{3}]$ an eta form on the base. Their construction was subsequently generalized in $[21]$ to situations where the family is not invertible, but admits a perturbation by a family $Q \in \Psi^{-\infty}(M / Y ; \mathbb{E})$ of self-ajoint smoothing operators such that $\partial+Q$ is invertible. The odd families index of the family $\partial$ is precisely the obstruction to the existence of such a family of perturbations; for the boundary operators of a family of Dirac-type operators on a fibration of manifolds with boundary, this index obstruction vanishes by the cobordism invariance of the index, so that invertible perturbations exist in this case.

When the family $\partial$ is invertible, the Bismut-Cheeger eta form is given by

$$
\eta_{\mathrm{BC}}(\widetilde{\partial})=\frac{1}{\sqrt{\pi}} \int_{0}^{\infty} \operatorname{STr}_{\mathbb{C} \ell(1)}\left(\frac{d \mathbb{B}_{t}}{d t} e^{-\mathbb{B}_{t}^{2}}\right) d t \in \Omega^{\text {even }}(Y),
$$

where $\mathbb{B}_{t}$ is the rescaled Bismut superconnection associated to $\partial$ (see (10.8) and (13.7) in [21] for a detailed discussion). For a family perturbed to be invertible, $\partial+Q$, the definition is slightly modified to

$$
\eta_{\mathrm{BC}}(ð+Q)=\frac{1}{\sqrt{\pi}} \int_{0}^{\infty} \operatorname{STr}_{\mathbb{C} \ell(1)}\left(\frac{d \widetilde{\mathbb{B}}_{t}}{d t} e^{-\widetilde{\mathbb{B}}_{t}^{2}}\right) d t \in \Omega^{\text {even }}(Y),
$$

where $\widetilde{\mathbb{B}}_{t}=\mathbb{B}_{t}+t^{\frac{1}{2}} \chi(t) Q \sigma$, with $\sigma \in \mathbb{C} \ell(1)$ a generator of $\mathbb{C} \ell(1)$ such that $\sigma^{2}=1$ and $\chi \in \mathcal{C}^{\infty}(\mathbb{R})$ is a non-negative function with $\chi(t)=0$ for $t<1$ and $\chi(t)=1$ for $t>2$. The Bismut-Cheeger eta form satisfies the following transgression formula.

Proposition 12. The exterior differential of $\eta_{\mathrm{BC}}(ð+Q)$ does not depend on the choice of perturbation $Q$ and is given by

$$
d \eta_{\mathrm{BC}}(ð+Q)=(2 \pi i)^{-\frac{n+1}{2}} \int_{M / Y} \hat{A}\left(R_{g}\right) \mathrm{Ch}^{\prime}(\mathbb{E}),
$$


where $n$ is the dimension of the fibres of the fibration $\phi: M \rightarrow Y$ and $\mathrm{Ch}^{\prime}(\mathbb{E})$ is the twisting Chern character of $\mathbb{E}$.

Proof. Because of the cut-off function $\chi$ we have that

$$
\begin{aligned}
\lim _{t \rightarrow 0} \frac{1}{\sqrt{\pi}} \operatorname{STr}_{\mathbb{C} \ell(1)}\left(e^{-\widetilde{\mathbb{B}}_{t}^{2}}\right) & =\lim _{t \rightarrow 0} \frac{1}{\sqrt{\pi}} \operatorname{STr}_{\mathbb{C} \ell(1)}\left(e^{-\mathbb{B}_{t}^{2}}\right) \\
& =\frac{1}{(2 \pi i)^{\frac{n+1}{2}}} \int_{M / Y} \hat{A}\left(R_{g}\right) \mathrm{Ch}^{\prime}(\mathbb{E}) .
\end{aligned}
$$

On the other hand, because the family $\partial+Q$ is invertible,

$$
\lim _{t \rightarrow \infty} \frac{1}{\sqrt{\pi}} \operatorname{STr}_{\mathbb{C} \ell(1)}\left(e^{-\widetilde{\mathbb{B}}_{t}^{2}}\right)=0
$$

exponentially fast. Thus, the result follows by combining (14.4) and (14.5) with

$$
\frac{d}{d t} \operatorname{STr}_{\mathbb{C} \ell(1)}\left(e^{-\widetilde{\mathbb{B}}_{t}^{2}}\right)=-d_{Y} \operatorname{STr}_{\mathbb{C} \ell(1)}\left(\frac{d \widetilde{\mathbb{B}}_{t}}{d t} e^{-\widetilde{\mathbb{B}}_{t}^{2}}\right)
$$

and integrating in $t$.

If we consider the rescaled version of the Bismut-Cheeger eta form,

$$
\widehat{\eta}_{\mathrm{BC}}(ð+Q)=\sum_{j=0}^{\infty}(2 \pi i)^{-j} \eta_{\mathrm{BC},[2 j]}(ð+Q),
$$

where $\eta_{\mathrm{BC},[2 j]}$ is the part of $\eta_{\mathrm{BC}}$ of degree $2 j$, then Proposition 12 shows that the Chern character of the family index is trivial in cohomology, which is consistent with the fact the odd index of $\partial$ must vanish for the invertible perturbation $\partial+Q$ to exist.

It also follows from Proposition 12 that if $Q_{1}$ and $Q_{2}$ are two perturbations giving invertible families, then $\widehat{\eta}_{\mathrm{BC}}\left(ð+Q_{1}\right)-\widehat{\eta}_{\mathrm{BC}}\left(\partial+Q_{2}\right)$ is a closed form. Moreover, using Proposition 12 again, it can be seen that the cohomolgy class represented by the form $\widehat{\eta}_{\mathrm{BC}}\left(ð+Q_{1}\right)-\widehat{\eta}_{\mathrm{BC}}\left(ð+Q_{2}\right)$ only depends on the homotopy classes of $Q_{1}$ and $Q_{2}$ in the space of such perturbations. This cohomology class can be identified usign the notion of spectral sections introduced in $[\mathbf{2 1}]$.

Definition 4. A spectral section for the family of self-adjoint Diractype operators $\partial$ of (14.1) is a family of self-adjoint projections $P \in \Psi^{0}(M / Y$; $\mathbb{E}$ ) such that for some smooth function $R: Y \rightarrow \mathbb{R}^{+}$(depending on $P$ ) and every $y \in Y$,

$$
\mathrm{\partial}_{y} u=\lambda u \Longrightarrow \begin{cases}P_{y} u=u, & \text { if } \lambda>R(y), \\ P_{y} u=0, & \text { if } \lambda<-R(y) .\end{cases}
$$


If $P_{1}$ and $P_{2}$ are spectral sections for the family $\precsim$, then as shown in [21], their formal difference defines a $K$-class $\left[P_{1}-P_{2}\right] \in K^{0}(Y)$. If $P_{1} P_{2}=P_{2}$, then $\left[P_{1}-P_{2}\right]$ is represented by the vector bundle given by the range of the family of the finite rank projections $\left(\mathrm{Id}-P_{2}\right) P_{1}$. In general, one can reduce to this case by choosing a third spectral section $R$ such that $P_{1} R=R, P_{2} R=R$ and setting

$$
\left[P_{1}-P_{2}\right]=\left[P_{1}-R\right]-\left[P_{2}-R\right] \in K^{0}(Y) .
$$

It is shown in $[\mathbf{2 1}]$ that such a spectral section $R$ always exists and that the definition of the $K$-class $\left[P_{1}-P_{2}\right]$ does not depend on the choice of $R$. To obtain a spectral section from an invertible self-adjoint perturbation $Q$, we need an extra assumption.

Definition 5. A family $Q \in \Psi^{-\infty}(M / Y ; \mathbb{E})$ of self-adjoint operators is spectrally finite with respect to the family of Dirac-type operators $\precsim$ if there exists a smooth function $R: Y \rightarrow \mathbb{R}^{+}$such that for every $y \in Y$,

$$
\partial_{y} u=\lambda u \Longrightarrow Q_{y} u=0 \text { if }|\lambda|>R(y) \text {. }
$$

If $Q \in \Psi^{-\infty}(M / Y ; \mathbb{E})$ is an invertible self-adjoint perturbation of the family $\widetilde{\partial}$ which is not spectrally finite, then using an approximation argument, it is shown in [21] that it is possible to deform it through invertible self-adjoint perturbations to one which is spectrally finite.

Suppose now that $Q \in \Psi^{-\infty}(M / Y ; \mathbb{E})$ is a spectrally finite invertible selfadjoint perturbation of the family $\partial$. Then there is a corresponding spectral section $P_{Q} \in \Psi^{0}(M / Y ; \mathbb{E})$ with $\left(P_{Q}\right)_{y}$ defined to be the projection onto the positive eigenspace of $\partial_{y}+Q_{y}$. The following relative index theorem was proved in $[\mathbf{2 1}]$.

Proposition 13. If $Q_{1}$ and $Q_{2}$ are two spectrally finite invertible selfajoint perturbations of the family $\mathrm{d}$, then the Chern character $\mathrm{Ch}\left(\left[P_{Q_{1}}-\right.\right.$ $\left.\left.P_{Q_{2}}\right]\right)$ of the $K$-class $\left[P_{Q_{1}}-P_{Q_{2}}\right] \in K^{0}(Y)$ is represented by the closed form

$$
\widehat{\eta}_{\mathrm{BC}}\left(ð+Q_{1}\right)-\widehat{\eta}_{\mathrm{BC}}\left(ð+Q_{2}\right) .
$$

If the odd index of the family $\partial$ does not vanish, it is still possible to define a version of the Bismut-Cheeger eta form, but now over an infinite dimensional bundle $\varpi: \mathcal{A}^{\mathrm{SA}} \longrightarrow Y$ defined in terms of self-adjoint smoothing perturbations. Namely the fibre at $y \in Y$ is

$$
\mathcal{A}_{y}^{\mathrm{SA}}=\left\{\widetilde{\partial}_{y}+Q_{y} ; Q_{y} \in \Psi^{-\infty}\left(Z_{y} ; \mathbb{E}\right), Q_{y}^{*}=Q_{y}, \widetilde{\partial}_{y}+Q_{y} \text { is invertible }\right\}
$$

The pull-back $\varpi^{*} \mathcal{A}^{\mathrm{SA}}$ of $\mathcal{A}^{\mathrm{SA}}$ to itself has a tautological section $\sigma_{\mathcal{A}^{\mathrm{SA}}}$ : $\mathcal{A}^{\mathrm{SA}} \longrightarrow \varpi^{*} \mathcal{A}^{\mathrm{SA}}$ which can be used to define a form on the total space of $\mathcal{A}^{\mathrm{SA}}$ via formula (14.3),

$$
\eta_{\mathrm{BC}} \in \Omega^{\mathrm{even}}\left(\mathcal{A}^{\mathrm{SA}}\right)
$$


It is well-defined since the space $\mathcal{A}^{\mathrm{SA}}$ has a natural structure of smooth Fréchet manifold. As in (14.6), we can also consider its rescaled version $\widehat{\eta}_{\mathrm{BC}} \in \Omega^{\text {even }}\left(\mathcal{A}^{\mathrm{SA}}\right)$. Proposition 12 then extends as follows.

Proposition 14. The exterior differential of the Bismut-Cheeger eta form $\eta_{\mathrm{BC}}$ in (14.9) is the basic form

$$
d \eta_{\mathrm{BC}}=\varpi^{*}\left((2 \pi i)^{-\frac{n+1}{2}} \int_{M / Y} \hat{A}\left(R_{g}\right) \mathrm{Ch}^{\prime}(\mathbb{E})\right) .
$$

Let $\rho \in \mathcal{S}(\mathbb{R})$ be a a choice of Schwartz function such that $\rho(0)=1$. Let also $\mathcal{A}$ be the infinite dimensional bundle of (5.1) with $A=\widetilde{\partial}$. The function $\rho$ can be used to define an injective bundle map $\iota: \mathcal{A}^{\mathrm{SA}} \rightarrow \mathcal{A}$ defined fibrewise by

$$
\iota_{y}: \mathcal{A}_{y}^{\mathrm{SA}} \ni \partial_{y}+Q_{y} \longmapsto \partial_{y}+i t+Q_{y} \rho(t) \in \mathcal{A}_{y} .
$$

The definition of $\iota$ clearly depends on the choice of $\rho$, but since the space of Schwartz functions equal to one at the origin is convex, the homotopy class of the map $\iota$ does not depend on the choice of $\rho$.

Proposition 15. For each $y \in Y$, the map $\iota_{y}: \mathcal{A}_{y}^{\mathrm{SA}} \longrightarrow \mathcal{A}_{y}$ is a weak homotopy equivalence; in particular, $\mathcal{A}_{y}^{\mathrm{SA}}$ is a classifying space for even $K$-theory and $\iota: \mathcal{A}^{\mathrm{SA}} \longrightarrow \mathcal{A}$ is also an homotopy equivalence.

Proof. Let $W \in \Psi^{-\infty}\left(Z_{y} ; \mathbb{E}_{y}\right)$ be a fixed choice of spectrally finite invertible self-adjoint perturbation of $\partial_{y}$. Let $B$ be a smooth closed manifold. Given a smooth map $f: B \longrightarrow \mathcal{A}_{y}^{\mathrm{SA}}$ with $f(b)=\partial_{y}+Q_{b}$, it is always possible to deform it through self-adjoint invertible perturbations so that the family $b \longmapsto Q_{b} \in \Psi^{-\infty}\left(Z_{y} ; \mathbb{E}_{y}\right)$ becomes spectrally finite with respect to $\partial_{y}$. This means there is a well-defined map

$$
\mu:\left[B ; \mathcal{A}_{y}^{\mathrm{SA}}\right] \ni[f] \longmapsto\left[P_{f}-P_{W}\right] \in K^{0}(B)
$$

where $P_{f}$ is the spectral section associated to the spectrally finite invertible self-adjoint perturbation $b \mapsto Q_{b}$ and $P_{W}$ is the spectral section associated to $W$ seen as a spectrally finite invertible perturbation for the trivial family $b \mapsto \partial_{y}$ over $B$. The map $\mu$ is easily seen to be bijective, so that $\mathcal{A}_{y}^{\mathrm{SA}}$ is a classifying space for even $K$-theory. On the other hand, under the identification

$$
\mathcal{A}_{y} \cong G_{\text {sus }}^{-\infty}\left(Z_{y} ; \mathbb{E}_{y}\right),
$$

given by composing on the right with $\left(\iota_{y}\left(\widetilde{\partial}_{y}+W\right)\right)^{-1}$, we see the fibre $\mathcal{A}_{y}$ is also a classifying space for even $K$-theory. In fact, this identification induces a map

$$
\nu:\left[B ; \mathcal{A}_{y}\right] \longrightarrow\left[B ; G^{-\infty}\left(Z_{y} ; \mathbb{E}_{y}\right)\right] \cong K^{-2}(B)
$$


and a corresponding commutative diagram

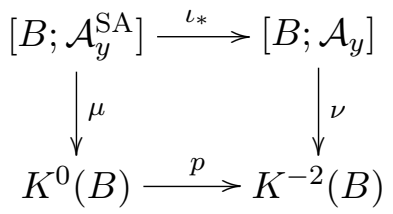

where the bottom map $p$ is Bott periodicity. In particular, this shows the map $\iota: \mathcal{A}_{y}^{\mathrm{SA}} \longrightarrow \mathcal{A}_{y}$ is a weak homotopy equivalence.

The proof of the previous proposition also gives the following result.

Corollary 2. Suppose that $\sigma_{1}: Y \rightarrow \mathcal{A}^{\mathrm{SA}}$ and $\sigma_{2}: Y \rightarrow \mathcal{A}^{\mathrm{SA}}$ are two sections of the bundle $\mathcal{A}^{\mathrm{SA}}$. Then the closed forms

$$
\left(\sigma_{1}^{*} \widehat{\eta}_{\mathrm{BC}}-\sigma_{2}^{*} \widehat{\eta}_{\mathrm{BC}}\right) \quad \text { and } \quad\left(\left(\iota \circ \sigma_{1}\right)^{*} \eta_{\mathcal{A}}-\left(\iota \circ \sigma_{2}\right) \eta_{\mathcal{A}}\right)
$$

represent the same cohomology class in $H_{\mathrm{dR}}^{\text {even }}(Y)$.

Proof. Using section $\sigma_{1}$ instead $W$ to define a map $\mu$ as in (14.11), we get a commutative diagram analogous to (14.13), namely,

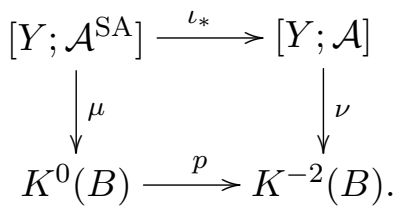

Thanks to Proposition 13, we then see that the forms $\left(\sigma_{1}^{*} \widehat{\eta}_{\mathrm{BC}}-\sigma_{2}^{*} \widehat{\eta}_{\mathrm{BC}}\right)$ and $\left(\left(\iota \circ \sigma_{1}\right)^{*} \eta_{\mathcal{A}}-\left(\iota \circ \sigma_{2}\right) \eta_{\mathcal{A}}\right)$ represent the Chern character of the same $K$-class, from which the result follows.

Since Proposition 15 shows that the bundles $\mathcal{A}^{\mathrm{SA}}$ and $\mathcal{A}$ are homotopy equivalent, the map $\iota$ allows $\eta_{\mathcal{A}}$ to be compared with the Bismut-Cheeger eta form $\widehat{\eta}_{B C}$. The definitions of $\eta_{\mathcal{A}}$ and $\widehat{\eta}_{\mathrm{BC}}$ involve different regularizations of the underlying Dirac family. Assuming choices of regularization and choices of connections affect the eta form in a similar way, it is natural in light of Lemma 4 to expect the following relation between the two types of eta forms.

Conjecture 1. There exist forms $\beta \in \Omega^{\text {even }}(Y)$ and $\alpha \in \Omega^{\text {odd }}\left(\mathcal{A}^{\mathrm{SA}}\right)$ such that

$$
\iota^{*} \eta_{\mathcal{A}}-\widehat{\eta}_{\mathrm{BC}}=\varpi^{*} \beta+d \alpha .
$$

As an indication that this conjecture might be true, we will prove it in a particular case.

THEOREM 4. The conjecture is true when the odd families index of the family $\precsim \in \Psi^{1}(M / Y ; \mathbb{E})$ vanishes. 
Proof. Since ind $(ð)=0$ in $K^{1}(Y)$, we know $\precsim$ admits an invertible self-adjoint perturbation $\partial+Q_{0}$ with $Q_{0} \in \Psi^{-\infty}(M / Y ; \mathbb{E})$. Without loss of generality, we can assume $Q_{0}$ is spectrally finite. The perturbation $Q_{0}$ defines a section $\sigma: Y \longrightarrow \mathcal{A}^{\mathrm{SA}}$ with $\sigma(y)=\mathrm{\partial}_{y}+\left(Q_{0}\right)_{y}$. There is also an induced section $\iota \circ \sigma: Y \longrightarrow \mathcal{A}$ for the bundle $\mathcal{A} \longrightarrow Y$. The form on the base is then taken to be

$$
\beta=\sigma^{*} \widehat{\eta}_{\mathrm{BC}}-(\iota \circ \sigma)^{*} \eta_{\mathcal{A}} .
$$

The form $\omega=\widehat{\eta}_{\mathrm{BC}}-\iota^{*} \eta_{\mathcal{A}}-\pi_{\mathcal{A}^{\mathrm{SA}}}^{*} \beta$ can then be written as the difference of two closed forms, $\omega=\omega_{\mathrm{BC}}-\omega_{\mathcal{A}}$ with

$$
\omega_{\mathrm{BC}}=\widehat{\eta}_{\mathrm{BC}}-\pi_{\mathcal{A}^{\mathrm{SA}}}^{*} \sigma^{*} \widehat{\eta}_{\mathrm{BC}}, \quad \omega_{\mathcal{A}}=\iota^{*} \eta_{\mathcal{A}}-\pi_{\mathcal{A}^{\mathrm{SA}}}^{*}(\iota \circ \sigma)^{*} \eta_{\mathcal{A}}
$$

Let $B$ be a closed manifold and $f: B \longrightarrow \mathcal{A}^{\mathrm{SA}}$ a smooth map. By perturbing $f$ in its homotopy class as necessary, it can be arranged that $f$ induces a spectrally finite invertible self-adjoint pertrubation of the family $f^{*} \partial$ parametrized by $B$. By Corollary 2 , both $f^{*} \omega_{\mathrm{BC}}$ and $f^{*} \omega_{\mathcal{A}}$ represents the Chern character of $\left[P_{f}-\left(\left(\pi_{\mathcal{A}^{\mathrm{SA}}} \circ f\right)^{*} P_{Q}\right]\right.$, where $P_{f}$ is the spectral section associated to the invertible family over $B$ defined by the map $f$ and $\left(\pi_{\mathcal{A}^{\mathrm{SA}}} \circ f\right)^{*} P_{Q}$ is the spectral section associated to the invertible family obtained by pulling back the invertible family $\partial+Q$ under the map $\pi_{\mathcal{A}^{\mathrm{SA}}} \circ f: B \longrightarrow Y$.

Since $B$ and $f$ are arbitrary, this means $\omega$ is trivial in the singular cohomology of $\mathcal{A}^{\mathrm{SA}}$. From the infinite dimensional version of the de Rham theorem in this context (see Lemma 6 below), it follows that there exists $\alpha \in \Omega^{\text {odd }}\left(\mathcal{A}^{\mathrm{SA}}\right)$ such that

$$
d \alpha=\omega,
$$

from which the result follows.

Lemma 6 (de Rham theorem). The de Rham theorem holds for the infinite dimensional space $\mathcal{A}^{\mathrm{SA}}$.

Proof. According to Theorem 16.10 and 34.7 in $[\mathbf{1 7}]$ or the discussion on p. 25 of $[\mathbf{2 9}]$, it suffices to show that $\mathcal{A}^{\text {SA }}$ satisfies the following two properties:

(i) the Fréchet space $\mathcal{F}$ on which $\mathcal{A}^{\mathrm{SA}}$ is locally modelled has enough smooth functions, which means that for each open set $\mathcal{U}$ in $\mathcal{F}$, there is a nonvanishing real-valued smooth function which vanishes outside $\mathcal{U}$;

(ii) The manifold $\mathcal{A}^{\mathrm{SA}}$ is Lindelöf, which means each open covering has a countable refinement.

To show property (i), fix $y \in Y$ and consider the Fréchet space $\Psi^{-\infty}\left(Z_{y} ; \mathbb{E}_{y}\right)$. Then the closed subspace

$$
\Psi_{\mathrm{SA}}^{-\infty}\left(Z_{y} ; \mathbb{E}_{y}\right)=\left\{Q \in \Psi^{-\infty}\left(Z_{y} ; \mathbb{E}_{y}\right) ; Q^{*}=Q\right\}
$$


is also naturally a Fréchet space. If $k=\operatorname{dim} Y$, then our local model for $\mathcal{A}^{\text {SA }}$ can be taken to be the Fréchet space

$$
\mathcal{F}=\Psi_{\mathrm{SA}}^{-\infty}\left(Z_{y} ; \mathbb{E}_{y}\right) \times \mathbb{R}^{k} .
$$

Since $\Psi^{-\infty}\left(Z_{y} ; \mathbb{E}_{y}\right)$ is a nuclear Fréchet space, so is $\mathcal{F}$ (see Corollary 21.6.4 and Corollary 21.2.3 in [16]). Thus, by Proposition 14.4 in [17], $\mathcal{F}$ has enough smooth functions.

To prove property (ii), notice that the Fréchet space $\Psi^{-\infty}\left(Z_{y} ; E_{y}\right)$ is separable, so it is in particular second-countable. This implies $\Psi_{\mathrm{SA}}^{-\infty}\left(Z_{y} ; \mathbb{E}_{y}\right)$ and $\mathcal{A}_{y}^{\mathrm{SA}}$ are second-countable, and more generally, that $\mathcal{A}^{\mathrm{SA}}$ is secondcountable, which means in particular it is Lindelöf.

\section{References}

[1] Pierre Albin and Richard Melrose, Relative Chern character, boundaries and index formula, Arxiv:0808.0183, August 2008.

[2] M. F. Atiyah, V. K. Patodi, and I. M. Singer, Spectral asymmetry and Riemannian geometry. I, Math. Proc. Cambridge Philos. Soc. 77 (1975), 43-69. MR 53 \#1655a.

[3] J-M Bismut and Cheeger J., $\eta$-invariants and their adiabatic limits, J. Amer. Math. Soc. 2 (1989), no. 1, 33-70.

[4] Jean-Michel Bismut and Jeff Cheeger, Families index for manifolds with boundary, superconnections and cones. II. The Chern character, J. Funct. Anal. 90 (1990), no. 2, 306-354. MR 91e:58181.

[5] _ Remarks on the index theorem for families of Dirac operators on manifolds with boundary, Differential geometry, Longman Sci. Tech., Harlow, 1991, pp. 59-83. MR 93k:58211.

[6] J.-L. Brylinski, Loop spaces, characteristic classes and geometric quantization, Progress in Mathematics, 107, Birkhäuser, Boston, MA, 1993.

[7] J.-L. Brylinski and D.A. McLaughlin, The geometry of degree-four characteristic classes and of line bundles on loop spaces. I, Duke Math. J. 75 (1994), no. 3, 603-638.

[8] U. Bunke, Transgression of the index gerbe, Manuscripta Math. 109 (2002), no. 3, 263-287.

[9] A. Carey and J. Mickelsson, A gerbe obstruction to quantization of fermions on odd dimensional manifolds, Lett. Math. Phys. 51 (2000), 145-160.

[10] - The Universal gerbe,Diximier-Douady classes and gauge theory, Lett. Math. Phys. 59 (2002), 47-60.

[11] A. Carey, J. Mickelsson, and M. Murray, Index theory, gerbes and hamiltonian quantization, Commun. Math. Phys. 183 (1997), 707-722.

[12] A. Carey, M.K. Murray, and B. Wang, Higher bundle gerbes and cohomology classes in gauge theories, J. Geom. Phys. 21 (1997), no. 2, 183-197.

[13] A. Carey and B. Wang, On the relationship of gerbes to the odd families index theorem, J. Geom. Phys. 57 (2006), no. 1, 23-38.

[14] K. Gawȩdzki and N. Reis, WZW branes and gerbes, Rev. Math. Phys. 14 (2002), 1281-1334.

[15] _ Basic gerbe over non-simply connected Lie groups, J. Geom. Phys. 50 (2004), no. $1-4,28-55$.

[16] H. Jarchow, Locally convex spaces, Teubner Stuttgart, 1981.

[17] A. Kriegl and P.W. Michor, The convenient setting of global analysis, American Mathematical Society, Providence, RI, 1997. 
[18] J. Lott, Higher-degree analogs of the determinant line bundle, Comm. Math. Phys. 230 (2002), no. 1, 41-69.

[19] E. Meinrenken, The basic gerbe on a compact simple Lie group, Enseign. Math. 49 (2003), no. 3-4, 307-333.

[20] Richard B. Melrose, The eta invariant and families of pseudodifferential operators, Math. Res. Lett. 2 (1995), no. 5, 541-561. MR 96h:58169.

[21] Richard B. Melrose and Paolo Piazza, Families of Dirac operators, boundaries and the b-calculus, J. Differential Geom. 46 (1997), no. 1, 99-180. MR 99a:58144.

[22] _ An index theorem for families of Dirac operators on odd-dimensional manifolds with boundary, J. Differential Geom. 46 (1997), no. 2, 287-334. MR 99a:58145.

[23] Richard B. Melrose and F. Rochon, Families index for pseudodifferential operators on manifolds with boundary, IMRN (2004), no. 22, 1115-1141.

[24] _ Boundaries, eta invariant and the determinant bundle, $\mathrm{C}^{*}$-algebras and elliptic theory II, Trends in Math, Birkhauser,149-181. (2006), 149-181.

[25] , Periodicity and the determinant bundle, Commun. Math. Phys. 274 (2007), 141-186.

[26] M.K. Murray, Bundle gerbes, J. London Math. Soc. 54 (1996), 403-416.

[27] M. Murray and D. Stevenson, The basic bundle gerbe on unitary groups, J. Geom. Phys. 58 (2008), no. 11, 1571-1590.

[28] S. Paycha and J. Mickelsson, Renormalized Chern-Weil forms associated with families of Dirac operators, Journal of Geometry and Physics 57 (2007), 1789-1814.

[29] A. Pressley and G. Segal, Loop groups, Oxford Mathematical Monographs, The Clarendon Press, Oxford University Press, New York, 1986.

[30] F. Rochon, Bott periodicity for fibred cusp operators, J. Geom. Anal. 15 (2005), no. 4, 685-722.

Department of Mathematics, Massachusetts Institute of Technology

E-mail address: rbm@math.mit.edu

Department of Mathematics, Australian National University

E-mail address: frederic.rochon@anu.edu.au 